Manuscript Number:

Title: Monocyte subsets in blood correlate with obesity related response of macrophages to biomaterials in vitro.

Article Type: FLA Original Research

Section/Category: Biomaterials and Regenerative Medicine (BRM)

Keywords: biomaterials; in vitro model; obesity; monocyte subsets; macrophages

Corresponding Author: Miss. Geesien S.A. Boersema, M.D.

Corresponding Author's Institution: Erasmus Medical Center

First Author: Geesien S.A. Boersema, M.D.

Order of Authors: Geesien S.A. Boersema, M.D.; Lizette Utomo; Yves Bayon; Nicole Kops; Erwin van der Harst; Johan F Lange; Yvonne M BastiaansenJenniskens

Abstract: Macrophages play a key role in the foreign body response. In this study it was investigated whether obesity affects the acute response of macrophages to biomaterials in vitro and whether this response is associated with biomarkers in blood. CD14+ monocytes were isolated from blood from obese and age and gender matched lean persons. Monocyte subsets were determined based on CD14 and CD16 on their surface. Creactive protein (CRP) was measured in peripheral blood. The response of monocyte-derived macrophages to polypropylene (PP), polylactic acid (PLA), polyethylene terephthalate (PET) monofilament, and PETmultifilament (mPET) in culture was based on cytokine production. More IL-6 (for PET), less CCL18 (all materials) and IL-1ra (for PLA) was produced by macrophages from obese patients than lean subjects. Body mass index, serum CRP and to a lesser extend percentages of monocyte subtypes correlated with IL-6, TNF $\alpha, \mathrm{CCL} 18$, and IL-1ra production. Taken together, monocyte-derived macrophages of obese patients respond more proinflammatory and less anti-inflammatory to biomaterials than macrophages from lean subjects, depending on the material. These results are a step towards personalized medicine for the development of a model or even a blood test to decide which biomaterial might be suitable for each patient. 
Rotterdam, The Netherlands, 27-06-2016

Dear professor Leong or whomever it may concern,

We are pleased to submit our revised manuscript entitled: 'Monocyte subsets in blood correlate with obesity related response of macrophages to biomaterials in vitro', again for consideration as an original article in Biomaterials. This manuscript provides an original research about different response of macrophages from obese and lean patients on different biomaterials that are common used for regenerative medicine.

Unfortunately, last time the manuscript could not be accepted, but encouraged by prof Grainger, who said that the work was of high quality and would be of interest to the biomaterials community, we adjusted the paper with the comments of the reviewers and add some more extra experiments, and a point-by-point response in this cover letter (and separately added in the submission).

This manuscript has not been previously published and is not under consideration in the same or substantially similar form in any other peer-reviewed media. We hope that the manuscript will now be considered for publication in Biomaterials.

On behalf of,

Y.M. Bastiaansen-Jenniskens, PhD (corresponding author)

y.bastiaansen@erasmusmc.nl

Department of Orthopedics

Sincerely,

G.S.A. (Simone) Boersema

Department of Surgery/ Orthopedics

Erasmus Medical Centre Rotterdam

g.boersema@erasmusmc.nl

phone nr. +31 (0)618342021 
Reviewer \#1:

Leptin, which is secreted by adipocytes, would be of interest as a parameter for obesity in addition to CRP. Please provide a rational for the selection of the clinical parameters in the introduction.

answer: We thank the reviewer for his/her suggestion to measure leptin. Our aim was to examine the effect of obesity on the macrophage-biomaterial interaction and whether this correlates to parameters in the blood in a clinical context. CRP was therefore chosen because it is indeed a standard clinical parameter for general inflammation and it was used as a standard measurement prior to surgery in our patient population, especially in people undergoing bariatric surgery. This is now also added to the introduction/materials and methods.

Experimental setup should be better described. The manuscript would benefit from a picture of the culture system, samples, and experimental approach.

answer: We apologize for being not clear in the experimental set up. A diagram is made to provide more clarity.

For the biomaterial study, yarn was used. From our experience achieving consistent seeding results on yarn or structured materials is difficult. How do the authors ensure that cells stayed on the biomaterial during culture and did not attached to the culture vessel? Please elaborate the description of the culture device and the seeding method.

answer: Before conducting the study with patient cells, pilot experiments were performed to determine the optimal material configuration allowing as much cells to be attached as possible. Disks, meshes and braided yarns (spool knit) were compared and the braided yarns resulted in the highest DNA amount directly after seeding and after culture. The braided materials are created of a mix of micro- and macro-porosity that favors cell attachment, particularly for monofilaments, and even more particularly, for polypropylene monofilaments. We did not make any effort to ensure cells would stay on the biomaterials. However, to ensure that medium is only conditioned by cells that adhered to the material and not by cells that adhered to the culture well, the yarns were replaced into new culture wells 24 hours prior to harvesting the culture medium. The culture system and seeding method is now included in the drawing that also depicts the experimental setup.

Furthermore, the cell surface ratio should be standardized for all materials. Otherwise interpretation of results is not possible. The currently used normalization on density does not consider the surface for cell attachment.

answer: All tested materials were braided according to a similar pattern and with same volumic density, corrected by the $\mathrm{g} / \mathrm{cm}^{3}$ values by each material. Since cell attachment was indeed different between materials, also because of different properties of the materials, we normalized the protein data to the amount of DNA as an indication for the cell number. We think that by normalizing for DNA, we adjust for variation in cell number allowing us to have a clear image of the production of cytokines per cell, not influenced by the number of cells that adhered to the material. More information on this is now provided in the materials and methods. 
Cytokine panel for characterization of pro- or anti-inflammatory effects is rather low. In general, higher numbers of different cytokines are used to characterize the material-cell interaction, e.g. Jaguin, M., et al. (2013) Cell Immunol 281(1): 51-61, Verreck, F. A. W., et al. (2006) Journal of Leukocyte Biology 79(2): 285-293.

answer: Indeed, very often, many cytokines are measured to examine the response of macrophages to biomaterials. However, we had a limited amount of culture medium due to the low number of macrophages available from the patients and healthy volunteers. In addition, in our previous studies, we saw that IL6, TNFalpha, IL1ra and CCL18 are the most discriminative for different phenotypes of macrophages $(1,2)$. Even though it is a rather small panel of cytokines, we think that this panel allows us to determine the phenotype and behavior of the macrophages in response to the material. The rationale behind our choice of cytokines is now more clearly explained in the Materials and Methods.

In the introduction, the authors describe that TNF-alpha and IL-6 is increased in obese people. This indicates, that data should be normalized to the base level of secretion without biomaterial contact. Thus, interpretation of the results is difficult.

answer: We thank the review for this remark. Indeed, obese people have increased circulating levels of TNFalpha and IL6, most likely produced by many types of cells than the macrophages alone. This is now clearer stated in the introduction. We do not agree however that data should be normalized to baseline levels because this is also not what happens in a clinical situation. Most likely, the fact that baseline (i.e. prior to implantation of a material) production is increased in some patients, contributes to the differences seen after implantation. In addition, even though corrections were not made for baseline levels, still differences are seen between the effect of biomaterials on cells of the same patient. This is now discussed in the discussion section.

The used cells for material incubation are not described and characterized regarding their macrophage identity. Although the monocytes are identified via CD-14 and CD-16, the differentiated macrophages are neither characterized nor differentiation is externally induced by growth factors. Characterization of biomaterial-adherent cells through immune histological staining should be conduct to confirm the macrophage M1/M2 index.

answer: We have extensive experience with macrophage culture, and after monocyte isolation and adherence to the culture substrate in the presence of $20 \%$ FCS, they differentiate into macrophages expressing the typical macrophage genes (1-4). We believe that by determining the cytokine production of TNFalpha, IL6, CCL18, and IL1ra we can determine the behavior and phenotype of the cells (1), rather than by determining the presence of cell surface markers that do not provide information on the behavior of the cells.

Following cell incubation, biomaterials are transferred to a 96-well plate. Thereby, nonadherent cells are excluded from the following investigations. Nevertheless, in vivo, the ecm as well as the cells in the proximity of the biomaterial contribute to the foreign body 
response. Please provide a rational for considering only the biomaterial-adherent cell fraction.

answer: We thank the reviewer for this interesting and valid point. The rationale behind transferring the materials with adherent cells to new wells, was to measure indeed only the cytokines that were produced by the adherent cells allowing us to determine the protein production in response the biomaterials of interest only not being overshadowed by the effect TCPS might have on the macrophages, which is in fact also a biomaterial that has an influence on macrophages. TCPS most likely will have a totally different effect than the extracellular matrix that normally surrounds an implanted biomaterial. In fact, we have seen that collagen indeed exerts different effect on macrophages than polymers $(2,3)$. We included the remark of this reviewer about the effect of the ECM and macrophages in the close proximity in the discussion and added our rationale behind only measuring the cytokine production of the adherent cells.

In our studies, we observed differences between thawed and fresh macrophages regarding the cluster of differentiation. Please add supplementary data that shows that CD-14 and CD-16 are unaffected by freezing and thawing.

answer: Indeed, cell death was observed after freezing and thawing. However, the percentages of monocyte subsets remained the same after thawing the cells again. We agree with the reviewer that more information on flow cytometric analysis should be given and we have now added plots of analyzed fresh cells and cells from the same donor that were frozen, also providing information on how measurements were done.

Usually, for an ELISA measurement $100 \mu 1$ of sample is required. In the culture device, the author's state that $125 \mu \mathrm{l}$ per test conditions is available. Conditions were provided in triplicates, which results in a total volume of $375 \mu 1$ per condition. Please explain how 4 different cytokines - requiring approximately $100 \mu \mathrm{l}$ - could be measured based on the limited sample volume. Are diluted concentration within the detection limit of the assays?

answer: We apologize for being unclear about this. Indeed, $125 \mu$ of culture medium was used per well, performed in triplicate. These triplicates were kept separately, allowing us to measure every condition and experiment in triplicate. Since from experience and pilot experiments we knew that certain materials induced certain levels of cytokines, we knew that medium needed to be diluted several times prior to measurement. Since every material resulted in different dilutions for the different cytokines, we did not include the dilution factors, because this would lead to 16 different dilutions (4 materials and 4 cytokines). However, to give the reviewer an idea, the dilutions ranged from $3 x$ to $100 x$, depending on the material and the cytokine. In addition, all measurements fitted within the standard curve, and if the value was too low, measurements were repeated with a lower concentration. More information is now provided in the methods section about the measurements of the cytokines. 
Cytokine concentrations in FCS may impact the experiment. Relevant controls (I) passive adsorption of measured proteins on material surface, and (II) concentration of IL-6. IL1ra, and TNF-alpha in FCS are missing. Measured cytokines usually can be found in the supplemented serum, and by binding to the surface, differences of cytokine concentrations could be explained by this effect. Measured cytokine concentrations should be interpreted in relation to blank medium following biomaterial incubation.

answer: We thank the reviewer for this remark and we agree that proper controls should be added. We now included a figure in which we determined the adsorption of our cytokines of interest to the materials and showing the values of these cytokines in our blank culture medium. As can be seen, our proteins of interest were not detectable in blank culture medium, and thus no adsorption was seen in blank culture medium. After spiking of these cytokines and chemokines however, adsorption was seen, and varying between the materials. Mostly, associations are made within a biomaterial. These comparisons and associations are therefore unaffected in our opinion. In figure 2, we are comparing materials with each other. Since PP had the most adsorption of our proteins of interest, the values for PP (and for PLA in the case of IL6) are most likely an underestimation. This is now added to the discussion.

Furthermore, the authors state that "the topography was not exactly the same", and thus the surface area was not comparable. Thus, differences of protein adsorption on different materials are increased and interpretation of results is difficult. Thus, measurements should be normalized to surface area.

answer: We now measured protein adsorption on the different materials and indeed, differences are seen in adsorption between materials. As mentioned in the answer above, most of the associations and comparisons are made within a biomaterial and a cytokine, not comparing two different cytokines or materials with each other, but comparing obese and lean patients, head and head, for all material cytokine combinations. These comparisons and associations are therefore unaffected in our opinion by the adsorption of the protein of interest. This is now added to the discussion. Specific area is hard to be evaluated due to i) stitches shadowing some possible areas, ii) stitches creating micro porosity which favors cell adhesion, iii) multifilament with theoretical much higher surface area, but real surface much reduced due to compaction of the filaments of the multifilament yarns. To minimize differences in surface area, the amount of materials incubated with cells were adjusted in such a way to get the same volume of material for each tested material PET, mPET, PP, and PLA.

Standard deviations of absolute protein production per individual for both groups (lean and obese people) are high and indicate that the readout depends on an unknown parameter that seems to exhibit an impact comparable to obesity. Please discuss.

answer: We agree with the reviewer that it is clear that not only obesity probably influenced the response of the macrophages to the biomaterials. We made an effort to rule out as many as possible other factors such as smoking, recent implantation of a biomaterial, diabetes mellitus or the use of immunosuppressive drugs but other unknown factors can still be of influence. This is now added to the discussion. 
Calculation of the M1/M2 index is rather unclear, even inconsistent. When comparing the methods and the results part, different definitions are given.

answer: We apologize for the apparent inconsistency. Accidentally the M2/M1 index was written in the materials and methods but this must be M1/M2 index as described in the results. We adjusted the description of the calculation hoping to be more clear in this.

There seems to be quite a lot of mathematics, and regarding the high standard deviations of the measured concentrations (Figure $2 \mathrm{~F}$ ) that are used to calculate the M1/M2 index, the small deviations depicted for the M1/M2 index is difficult to understand.

answer: For every cytokine, normalization was performed to the average of that cytokine level in the response to the biomaterial. Therefore, standard deviations are small. We hope that by explaining the calculation of the M1/M2 index in more detail, this is made clearer.

In contrast to CCL18, IL-6 is secreted in a 100-fold higher range, and thus weighting in the M1/M2 index is biased by cytokines exhibiting higher concentration ranges. Thus, concentrations should be normalized and scaled.

answer: Indeed, for the calculation of the M1/M2 index, a normalization is performed for every cytokine to its average within the measurements for that biomaterial. This prevents that a cytokine that is produced at higher levels has more influence on the index than a cytokine that is produced at lower levels. We hope that our earlier adjustments made this more clear.

I appreciate the personalized-medicine-aligned study approach that considers health factors of individual patients. Furthermore, to aim at a blood test that facilitates the identification of biomaterials suitable for a specific patient is of great interest. However, study design requires further controls to allow a sound characterization of the biomaterial-patient interaction. Furthermore, test conditions are not standardized (cell identity, surface area). My recommendation is to reject the manuscript.

answer: We thank the reviewer for his appreciation for our personalized-medicinealigned study approach and we hope that by addressing his comments in detail, made adjustments, and added controls, this reviewer now thinks our paper is ready for publication.

Reviewer \#2:

This is a rather phenomenological, and it is unclear if the in vitro results really represent what happens in vivo. However, the presented results open an interesting scenario worth to investigate and discuss among the scientific community, with potential impact on personalized treatments after surgery and in regenerative medicine. Therefore, this article merits consideration by biomaterials audience with some revisions to improve the quality of the manuscript

answer: We thank the reviewer for pointing out the relevance of our paper. We agree that results should not be over interpreted but that our study opens up 
interesting perspective for future research and application. Therefore we adjusted a few of our sentences in the conclusions section.

1. In the abstract authors claim: " These results are a step towards personalized medicine for the development of a model or even a blood test to decide which biomaterial might be suitable for each patient." I disagree with the authors. There is no clear material-specific differences among the materials tested. There is a difference between obese and lean, and in most data the trends are similar among the tested materials.

answer: In supplementary former figure $2 \mathrm{~F}$, comparisons are made between the cytokine levels in response to the different materials. From this graph, we concluded that there are indeed different responses in the macrophages in response to the different biomaterials. In addition, as this reviewer points out later, differences are seen on the M1/M2 index that is based on all these cytokines. We now made this figure one of the main figures, since a part of our conclusion is based on these results.

2. In each figure (in the manuscript and in the supporting information) the number of different samples ("n") used for the experiments should be specified. This has only been done for some figures and is relevant to assess the significance of the results (authors say that they discharged certain subpopulations for the different tests)

answer: We thank the reviewer for pointing this out and added this information to all the figures.

3. The gender distribution among the samples was male/female $2 / 18$. This should be specified not only on Table 1 but also in the experimental part.

answer: We agree with the reviewer and added this information to the plots in which individual measurements are shown (supplementary figure 2A-D). Since the distribution of male/female is different for every material and measured cytokine, we choose not to add it to the other figures, because this would decrease the clarity of the figures.

4. Figure 1: M1/M2 index between lean and obese patients changes is much larger on mPET than on PET, although it is the same material. Author should comment on this.

answer: This is indeed an interesting point raised by the reviewer and it shows that not only the polymer itself is important for the response of the macrophages to the material, but also the architecture of the material. This is now clearer discussed in the discussion section.

1. Utomo L, van Osch GJ, Bayon Y, Verhaar JA, Bastiaansen-Jenniskens YM. Guiding synovial inflammation by macrophage phenotype modulation: an in vitro study towards a therapy for osteoarthritis. Osteoarthritis Cartilage. 2016 Apr 14.

2. Grotenhuis N, Bayon Y, Lange JF, Van Osch GJ, Bastiaansen-Jenniskens YM. A culture model to analyze the acute biomaterial-dependent reaction of human primary macrophages. Biochem Biophys Res Commun. 2013 Mar 29;433(1):115-20. 3. Grotenhuis N, Vd Toom HF, Kops N, Bayon Y, Deerenberg EB, Mulder IM, van Osch GJ, Lange JF, Bastiaansen-Jenniskens YM. In vitro model to study the 
biomaterial-dependent reaction of macrophages in an inflammatory environment. Br J Surg. 2014 Jul;101(8):983-92.

4. Fahy N, de Vries-van Melle ML, Lehmann J, Wei W, Grotenhuis N, Farrell E, van der Kraan PM, Murphy JM, Bastiaansen-Jenniskens YM, van Osch GJ. Human osteoarthritic synovium impacts chondrogenic differentiation of mesenchymal stem cells via macrophage polarisation state. Osteoarthritis Cartilage. 2014 Aug;22(8):1167-75. 


\title{
Monocyte subsets in blood correlate with obesity related response of macrophages to biomaterials in vitro.
}

G.S.A. Boersema ${ }^{a, b}$, L. Utomo ${ }^{b}$, Y. Bayon ${ }^{c}$, N. Kops ${ }^{b}$, E. van der Harst ${ }^{d}$, J.F. Lange ${ }^{a}$, Y.M. BastiaansenJenniskens ${ }^{b}$

${ }^{a}$ Department of Surgery, Erasmus MC, University Medical Center Rotterdam, The Netherlands

${ }^{b}$ Department of Orthopaedics, Erasmus MC, University Medical Center Rotterdam, The Netherlands 'Medtronic-Sofradim production, 116 Avenue Formans, 01600 Trévoux, France

${ }^{d}$ Department of Surgery, Maasstad Hospital, Rotterdam, the Netherlands

\author{
Corresponding author \\ Y.M. Bastiaansen-Jenniskens, PhD \\ y.bastiaansen@erasmusmc.nl \\ Department of Orthopedics \\ Wytemaweg 80, Ee1651b, 3015 GE Rotterdam \\ Erasmus MC, University Medical Center \\ Tel: +31-10-7044626 \\ Fax: +31-10-7044690
}




\begin{abstract}
Macrophages play a key role in the foreign body response. In this study it was investigated whether obesity affects the acute response of macrophages to biomaterials in vitro and whether this response is associated with biomarkers in blood. CD14+ monocytes were isolated from blood from obese and age and gender matched lean persons. Monocyte subsets were determined based on CD14 and CD16 on their surface. C-reactive protein (CRP) was measured in peripheral blood. The response of monocyte-derived macrophages to polypropylene (PP), polylactic acid (PLA), polyethylene terephthalate (PET) monofilament, and PET-multifilament (mPET) in culture was based on cytokine production. More IL-6 (for PET), less CCL18 (all materials) and IL-1ra (for PLA) was produced by macrophages from obese patients than lean subjects. Body mass index, serum CRP and to a lesser extend percentages of monocyte subtypes correlated with IL-6, TNF $\alpha$, CCL18, and IL-1ra production. Taken together, monocyte-derived macrophages of obese patients respond more pro-inflammatory and less anti-inflammatory to biomaterials than macrophages from lean subjects, depending on the material. These results are a step towards personalized medicine for the development of a model or even a blood test to decide which biomaterial might be suitable for each patient.
\end{abstract}

Keywords: biomaterials, in vitro model, obesity, monocyte subsets, macrophages 


\section{Introduction}

Biomaterials are often used in several surgical disciplines such as urology, gynaecology and general surgery[1]. The foreign body response to implanted biomaterials is crucial for adapting the material in the human body. Macrophages play a key role in the foreign body reaction to biomaterials[2]. For regenerative biomaterials, an initial pro-inflammatory (M1) response is necessary for recruiting inflammatory cells to encourage the foreign body response, which are necessary events for wound healing including ingrowth. However, a prolonged M1 response results in fibrous capsule formation and extended inflammation. Therefore, a subsequent transition to the anti-inflammatory macrophages (M2), which promotes tissue repair and remodeling, is generally presumed to be the preferred modification[3]. Achieving the desired outcome is individual and biomaterial dependent.

In general, obesity seems to be an important factor for adverse outcomes after surgery. Observed complications are surgical site infections, impairment of cutaneous wound healing, wound failure, anastomotic leakage, and fascia dehiscence[4-6]. These complications are major risk factors to develop incisional hernia or a recurrent incisional hernia after repair[7, 8]. Potential factors that increase wound complications by obesity include intrinsic tenuous anatomic properties, poor vascularization, and cellular and molecular alterations. Inflammatory mediators such as tumor necrosis factor alpha (TNF $\alpha$ ), interleukin 6 (IL-6), leptin, and angiotensin increase simultaneously with increasing mass of adipose tissue and adipocyte size[4]. These factors negatively affect wound healing and are most likely produced by many types of cells than macrophages alone. Obesity is also positively correlated with oxidative stress which can lead to decreased oxygen tension and impaired fibroblast proliferation and collagen synthesis[4].

Due to obesity, macrophages undergo a phenotypic switch from $M 2$ to $M 1$, which leads to a chronic low-grade systemic inflammation[9-13]. Monocytes, the precursors of macrophages, can be divided into subsets, according to their expression of the cell surface antigens CD16 (Fcy receptor III) and CD14 (a receptor for bacterial lipopolysaccharide (LPS))[14]. The classical monocyte has high CD14 $\left(\mathrm{CD} 14^{++}\right)$cell surface expression and is CD16 negative (CD16). The non-classical monocyte also expresses CD14 at its surface but at an approximately ten times lower level than the classical monocyte $\left(\mathrm{CD} 14^{+}\right)$, and is positive for $\mathrm{CD} 16\left(\mathrm{CD} 16^{++}\right)$. The intermediate monocyte expresses $\mathrm{CD} 14$ at a high level $\left(\mathrm{CD} 14^{++}\right)$, and $\mathrm{CD} 16$ at an approximately ten times lower level than the non-classical monocyte $\left(\mathrm{CD} 16^{+}\right)$. In general, monocytes expressing $\mathrm{CD} 16$ have a high phagocytic capacity and produce more pro-inflammatory cytokines such as TNF $\alpha$ and IL-6, and are therefore considered proinflammatory [15]. The classical $\mathrm{CD} 14^{++} / \mathrm{CD} 16^{-}$subset is the predominant population and accounts for 
approximately $90 \%$ in healthy persons. It has been suggested that obesity leads to a shift from classical towards intermediate and non-classical monocytes[16, 17].

Previous in vitro models have shown that culturing macrophages isolated from healthy donors on different biomaterials leads to a biomaterial-specific reaction[18] and that even in a contaminated in vitro model, surgical biomaterials still elicit differential reactions in macrophages[19]. These in vitro models did not take into account patient specific characteristics, such as age, smoking, diabetes or obesity. Obesity is a growing healthcare issue in the clinics and a subgroup of these patients does receive a biomaterial for several reasons like abdominal wall hernia with an increased risk of unwanted reactions to the biomaterial or delayed wound healing $[4,5]$. Therefore, the aims of this study were to investigate how obesity affects the acute host response of macrophages to biomaterials in vitro and to examine whether this in vitro response can be predicted beforehand by determining monocyte subsets in the blood or by measuring the systemic inflammation marker CRP that is a common used clinical parameter for inflammation. 


\section{Methods}

\section{Study population}

In total we included 20 obese patients and 20 age and gender matched healthy, lean (BMI 18-25 $\mathrm{kg} / \mathrm{m}^{2}$ ) volunteers. Obese patients with a BMI $>30 \mathrm{~kg} / \mathrm{m}^{2}$ were included at the department of bariatric surgery at the Maasstad Hospital, Rotterdam. Exclusion criteria for both groups were smoking, diabetes mellitus, use of immunosuppressive drugs, autoimmune disease or chronic inflammatory disease, and medical history such as previous surgery or having a prosthesis (e.g. vascular implants, mesh, osteosynthesis material). This study was approved by the Medical Ethical Committee of the Erasmus University Medical Center, Rotterdam, Netherlands, in accordance with the Dutch law on medical research in humans. Permit number MEC-2014-221, NL47780.078.14. Written informed consent was obtained from all patients.

\section{Biomaterials}

Four types of biomaterials were selected for use in all experiments: polypropylene (PP; $0.9 \mathrm{~g} / \mathrm{cm}^{3}$ ), polyethylene terephthalate multifilament (MPET; $1.34 \mathrm{~g} / \mathrm{cm}^{3}$ ), polyethylene terephthalate monofilament (PET; $1.34 \mathrm{~g} / \mathrm{cm}^{3}$ ) and polylactic acid (PLA; $1.25 \mathrm{~g} / \mathrm{cm}^{3}$ ) (Figure 1). All materials were provided as yarns braided in the same conformation. All tested materials were braided according to a similar pattern and with same volumic density, corrected by the $\mathrm{g} / \mathrm{cm} 3$ values by each material. The braided materials are created of a mix of micro- and macro-porosity that favors cell attachment, particularly for monofilaments, and even more particularly, for polypropylene monofilaments.

\section{Monocyte isolation and seeding on biomaterials}

Peripheral blood mononuclear cells (PBMC) were isolated from $30 \mathrm{~mL}$ blood of obese patients and healthy volunteers by gradient density separation using Ficoll-Paque PLUS (GE Healthcare Life Sciences, Little Chalfont, UK). The blood from the obese patients was obtained preoperatively to bariatric surgery. Monocytes were isolated by $\mathrm{CD}_{14}{ }^{+}$selection. Briefly, the blood was diluted 1:1 with PBS (Gibco; Carlsbad, USA) supplemented with 0.1\% BSA (Sigma-Aldrich, St. Louis, USA), applied on top of a Ficoll layer, and centrifuged at $900 \times g$ for 30 minutes to acquire separation of layers. The interphases were collected and washed twice with PBS/0.1\% BSA before a 20 minute incubation at $4^{\circ} \mathrm{C}$ with anti-human CD14 MicroBeads (Miltenyi Biotec, Bergisch Gladbach, Germany). Positive selection was performed by Magnetic-acitvated cell sorting (MACS). The isolated CD14 ${ }^{+}$monocytes were kept in suspension in X-VIVO ${ }^{\mathrm{TM}} 15$ medium (Lonza, Verviers, Belgium) containing $20 \%$ heat inactivated fetal calf serum (FCS; Lonza), $50 \mu \mathrm{g} / \mathrm{mL}$ gentamicin (Gibco) and $1.5 \mu \mathrm{g} / \mathrm{mL}$ amphotericin B (Fungizone; Gibco), from now on referred to as 'culture medium', until seeding. Prior to seeding, the biomaterials were pre-conditioned in non-heat inactivated FCS for 2 hours at $37^{\circ} \mathrm{C}$ with agitation. 
After pre-conditioning, the monocytes were seeded by rotation onto the biomaterials for 2 hours at

\section{Protein adsorption by the biomaterials}

To evaluate potential adsorption of the proteins by the materials, the materials were pre-conditoned for 2 hours in non-heat inactivated FCS with agitation, followed by 2 hours incubation in X-VIVO/20\% $\mathrm{FCS}$ in a tube rotator at $37^{\circ} \mathrm{C}$. Next, the materials were transferred to well plates and incubated in XVIVO/20\% FCS for 2 days. After this period, the materials were transferred to new well plates and incubated in medium containing either $1 \mathrm{ng} / \mathrm{mL}$ IL-6 (Peprotech), $250 \mathrm{pg} / \mathrm{mL}$ CCL18 (R\&D Systems), $1.25 \mathrm{ng} / \mathrm{mL}$ IL 1RA (R\&D Systems), $500 \mathrm{pg} / \mathrm{mL}$ TNF $\alpha$ or no cytokine. After an additional incubation day, the media were harvested, centrifuged at $300 \times \mathrm{g}$ and stored at $-80^{\circ} \mathrm{C}$ until cytokine quantification. The use dosages were based on the detection ranges of the enzyme-linked immunosorbent assays (ELISAs) that were used to determine cytokine concentrations.

\section{Cytokine quantification}

Enzyme-linked immunosorbent assays (ELISAs) were performed according to manufacturer's instructions to quantify the concentrations of CCL18, IL-1ra, IL-6, and TNF $\alpha$ (R\&D Systems, Minneapolis, MN, USA) released in the cell culture supernatants. These selected cytokines were chosen based on our previous research in which CCL18, IL-1ra, IL-6, and TNF $\alpha$ were the most discriminative for the different macrophages phenotypes $[18,20]$. All measurements fitted within the standard curve, for every material and cytokine different dilutions had to be made of the culture medium, ranging from a 3 to 100 times dilution. C-Reactive Protein (CRP) levels in the plasma were determined using the standard technique at the hospital's laboratory (Dimension Vista ${ }^{\circledR}$ System, Flex reagent cartridge, Siemens Healthcare Diagnostics Products, Germany) and expressed in mg/L. CRP is 
a very common used parameter in all hospitals to detect early systemic inflammation, also prior to surgery, especially in obese patients.

\section{DNA quantification}

Since cell attachment was different between materials we normalized the protein content in the culture media to the amount of DNA on the biomaterial as an indication for the cell number. By normalizing for DNA, we adjust for variation in cell number allowing to determine the production of cytokines per cell, not influenced by the number of cells that adhered to the material. DNA was quantified with a modified CYQUANT ${ }^{\circledR}$ cell proliferation assay (Invitrogen, Carlsbad, California, USA), in order to normalize the cytokine production for the number of cells. In short, the samples were sonicated for 30 minutes at $48 \mathrm{kHz}$ to completely disintegrate the cells. Next, a solution containing 250 IU heparin (LEO Pharma, Ballerup, Denmark) and $125 \mu \mathrm{g}$ RNAse (Sigma-Aldrich) were added to the suspensions and incubated for 30 minutes at $37^{\circ} \mathrm{C}$. Finally, $0.375 \mu \mathrm{L}$ CyQUANT GR dye was added to each sample and fluorescence was immediately measured on a SpectraMax Gemini micro plate reader (Molecular Devices, Sunnyvale, USA) at $480 \mathrm{~nm}$ excitation and $520 \mathrm{~nm}$ emission.

\section{Flow cytometric analysis}

Monocytes were thawed from $-80^{\circ} \mathrm{C}$ and re-suspended at 500,000 cells $/ \mathrm{mL}$ in FACSFlow solution (BD Biosciences) and stained for 30 minutes at $4^{\circ} \mathrm{C}$ with antibodies against human CD14 conjugated with APC-H7 and CD16 conjugated with PE (both BD Biosciences), according to the manufacturer's guidelines. Unstained cells were used as negative control. Flow cytometric analysis was performed using the FACSJazz ${ }^{\mathrm{TM}}$ (BD Biosciences), and data were analyzed using FlowJo (FlowJo v7.6.4/v10; Ashland, OR, USA). As can be seen in supplementary Figure 1, cells changed in shape and granularity ( $A$ and $C$ ), and most likely because of cell death, less cells were stained with either of the two antibodies after freezing and thawing. Percentages of monocytes subsets remained however comparable (B and D).

\section{Statistical analysis}

Statistical analysis was performed with SPSS 21.0 (IBM Inc., Chicago, USA). Basic characteristics are presented as mean \pm standard deviation (SD) and data related to cytokines are presented as mean and standard of mean (S.E.M.). An independent T-test was used for the age and BMI due to normal distribution of these parameters. Mann Whitney $U$ analysis was used for statin use. To compare cytokine levels between macrophages obtained from lean and obese subjects and compare cytokine levels between the different materials within the obese and control group, a Kruskal-Wallis analysis followed by a post-hoc Mann Whitney $\mathrm{U}$ analysis was performed. An M1/M2-index per material was 
calculated based on the cytokine production of pro-inflammatory (M1) cytokines IL-6 and TNF $\alpha$ and anti-inflammatory (M2) cytokines CCL18 and IL-1ra. The mean of the relative M1 cytokine levels per patient to the overall M1 cytokine levels of all patients, was divided over the mean of the relative M2 cytokine levels per patient to the overall M2 cytokine production of all patients, as shown in the following formula.

$$
M 1 / M 2-\text { index }=\frac{\frac{\left(\mathrm{IL}-6_{\text {per patient }}+\mathrm{TNF} \alpha_{\text {per patient }}\right)}{\left(\overline{\mathrm{IL}-6_{\text {all patients }}}+\overline{\left.\mathrm{TNF} \alpha_{\text {all patients }}\right)}\right.}}{\frac{\left(\mathrm{CCL} 18_{\text {per patient }}+\mathrm{IL} 1 \mathrm{ra}_{\text {per patient }}\right)}{\left(\overline{\mathrm{CCL} 18_{\text {all patients }}}+\overline{\left.\mathrm{IL} 1 \mathrm{ra}_{\text {all patients }}\right)}\right.}}
$$

To determine correlations, a non-parametric Spearman test was performed. All reported $p$-values were two-sided; a $p$-value $<0.05$ was considered to indicate statistical significance. Since the analyses were exploratory and the groups sizes small, no adjustment for multiple testing was performed. 


\section{Results}

As a result of our inclusion criteria, BMI was significantly different between the included lean and obese subjects. Age, gender and the use of statins were not significantly different between the two groups (Table 1).

\section{Obesity influenced cytokine production by macrophages on biomaterials}

The production of IL-6 and TNF $\alpha$ as indicators for a pro-inflammatory response and CCL18 and IL-1ra as indicators for an anti-inflammatory response were measured. Macrophages from obese patients produced significantly more IL-6 than macrophages from lean subjects when cultured on PET (144.0 pg IL-6/ng DNA vs 102.0 pg IL-6/ng DNA, $p=0.022$ ). No significant differences were seen for the other materials regarding IL-6 production (Table 2 and Supplementary Figure 2A). TNF $\alpha$ production was not significantly different between the groups for any of the tested materials (Table 2 and Supplementary Figure 2B). CCL18 production was significantly higher for all materials in the lean group than in the obese group (Table 2 and Supplementary Figure 2C). IL-1ra production was higher in the lean group than in the obese group when cultured on PLA (34.6 pg IL-1ra/ng DNA vs 15.5 pg IL$1 \mathrm{ra} / \mathrm{ng} \mathrm{DNA}, p=0.026$ ) but not on the other materials (Table 2 and Supplementary Figure 2D).

No IL-6, TNFa, CCL18, and IL-1ra were detectable in medium with serum alone and thus also no difference was seen after incubation of the material in medium with serum but without adherent cells. When the proteins of interest were spiked in the culture medium, adsorption of these proteins was seen to the materials, with the most adsorption of all four proteins to PP, and PLA in the case of IL-6 (Figure 2).

The DNA concentration as an indication for the number of attached macrophages to the biomaterials, was not significantly different between the lean and obese patients in all biomaterials (Supplementary data Figure 3A). Absolute protein production per individual is shown in supplementary Figure 2B-D. When comparing the effect of the materials on macrophages within the obese and lean group and per material, PP induced higher levels of IL-6, TNF $\alpha$, and IL-1ra than the other materials, especially when compared to MPET. Less clear differences between materials were seen for CCL18 production (Figure 3).

To compare overall response of the different materials in lean and obese subjects, an M1/M2 index was calculated for each condition. The $M 1 / M 2$ index was significantly higher of the obese group than for the lean subjects for PP $(p<0.001)$, PET $(p=0.001)$, and MPET $(p=0.003)$ but not for PLA. No differences regarding the M1/M2 index were seen between materials for the lean subjects. In obese patients, PLA resulted in the lowest M1/M2 index, and mPET the highest (Figure 4). 
Serum CRP and BMI correlate with cytokine production by macrophages

The average C-reactive protein level in lean subjects was $1.3 \pm 1.8 \mathrm{mg} / \mathrm{L}$ versus $15.6 \pm 17.1$ $\mathrm{mg} / \mathrm{L}$ in obese patients, $p=0.004$ (Supplementary Figure 4). CRP concentration positively correlated with BMI (Table 3). A positive correlation was also seen between CRP and IL-6 production in response to the material for all materials, but only significant for PP and MPET. A significant negative correlation was seen between serum CRP concentration and CCL18 production by macrophages in response to $\mathrm{PP}, \mathrm{PLA}$, and $\mathrm{mPET}$ and between $\mathrm{BMI}$ and CCL18 production by macrophages in response to PP, PLA, and mPET. CRP also negatively correlated with IL-1ra production in response to PP, PLA, and $\mathrm{MPET}$. CRP or BMI did not correlate with TNF $\alpha$ production (Table 3).

\section{Differences in monocyte subsets between lean and obese patients}

The percentages of classical monocytes $\left(\mathrm{CD} 14^{++} \mathrm{CD} 16\right)$, intermediate monocytes $\left(\mathrm{CD} 14^{++} \mathrm{CD} 16^{+}\right)$, or non-classical monocytes $\left(\mathrm{CD} 14^{+} \mathrm{CD} 16^{++}\right)$in peripheral blood were not statistically significantly different between lean and obese subjects (Table 4). However, the percentages of intermediate monocytes correlated positively with IL-6 for PLA and negatively with the CCL18 protein production for PET and mPET, and with IL-1ra for mPET. The percentages of non-classical monocytes correlated negatively with CCL18 production when macrophages were cultured on MPET. No statistically significant correlations were seen between percentages of monocyte subsets and TNF $\alpha$ production by the macrophages cultured on any of the biomaterials (Table 5A). For PP and mPET the $M 1 / M 2$ index significantly correlated with the percentage of classical monocytes. Intermediate monocytes significantly correlated negative with the M1/M2 index for mPET. Supplementary Figure 1 shows that the percentages of monocyte subsets are unaffected before and after thawing. 


\section{Discussion}

The use of biomaterials has become common in regenerative medicine. The reaction of primary human macrophages to biomaterials has been shown in vitro to be biomaterial specific, even when an inflammatory situation is simulated $[18,19]$. However, the person-dependent foreign body response has not been taken into account in these models. In the current explorative study, we investigated the effect of obesity, a growing problem in the Western world, on the response of macrophages to biomaterials and found that on average macrophages from obese patients respond more pro-inflammatory to biomaterials as indicated by higher IL- 6 and lower CCL18 and IL-1ra production than in macrophages from lean persons that were cultured on the same materials. In addition, we found that BMI, serum CRP and percentages of monocyte subsets in the peripheral blood correlate with the response of the macrophages to the biomaterials in vitro, and that these correlations were biomaterial specific. In addition, we showed that macrophages derived from monocytes from obese patients still respond pro-inflammatory, even when they are not in an obese environment anymore. To our knowledge, this is the first study that investigated the differences in macrophage response to biomaterials between lean and obese patients.

Obese patients included in this trial had no insulin resistance and therefore, according to the WHO criteria, no metabolic syndrome[21]. Because of the strict inclusion and exclusion criteria, such as no smoking, no implants, and the absence of diabetes mellitus we assume that the different responses to the biomaterials between lean and obese patients is the result of obesity only and not because of a difference in the presence of diabetes or previous operations in which biomaterials were used. However, certain risk factors that are unknown at the moment might have influenced our measurements and have resulted in the large variation that is sometimes observed in the cytokine measurements. Although these patients do not have a metabolic syndrome, 50\% of them had a CRP level $>10 \mathrm{mg} / \mathrm{L}$, indicating systemic low-grade inflammation. CRP levels in the serum correlated positively with IL-6 production by the macrophages on PP and MPET and negatively with CCL18 and IL-1ra levels on PP, PLA and MPET in vitro, showing that CRP has a pro-inflammatory effect on macrophages. This was supported by an in vitro study, where it was shown that CRP polarizes human macrophages to an M1 phenotype[22]. A shift from classic monocytes in the peripheral blood to intermediate or non-classic monocytes has been seen before as a result of obesity $[11,23,24]$, of which the latter two subsets are regarded as the pro-inflammatory subsets with increasing CD16 positivity[11, 23, 24]. We did not observe a statistically significant shift when comparing the presence of these subsets between lean persons and obese patients. This can be due to the fact that the inclusion criteria were strict and only obese patients without a metabolic syndrome were included. In addition, the numbers of patients from who we were able to obtain a sufficient number of 
monocytes to perform additional flow cytometric analysis next to culture with biomaterials were low subsets in the peripheral blood with the cytokine production of monocyte-derived macrophages on biomaterials in vitro, CCL18 and IL-1ra production by macrophages on MPET and PET in vitro were correlated with the percentages of the different monocyte subsets in the peripheral blood. The percentages of classical monocytes correlated positively with CCL18 and IL-1ra levels produced by macrophages in culture, the percentages of the more pro-inflammatory intermediate and nonclassical subsets correlated negatively with CCL18 production in culture. CCL18 is a chemokine that is predominantly made by anti-inflammatory macrophages $[18,25]$, indicating that the initial presence of classical monocytes is associated with the differentiation towards anti-inflammatory macrophages. As could be seen from the individual levels of IL-6, CCL18 and IL-1ra, not all obese patients had macrophages producing high levels of IL- 6 and low levels of CCL18 or IL-1ra. No corrections for baseline production of the cytokines of interest were made however, because in our opinion, this best represents the in vivo situation. Even though no corrections were made, differences were still seen between the effects of different biomaterials on cells of the same patient. This underlines potential patient specific responses even when obesity already changed the metabolic status of the patient and these responses can be explained by serum CRP levels and percentages of monocyte subsets in the blood. The production of TNFa in our culture system was not influenced by obesity, this might be explained by the short time detection range of TNF $\alpha[26]$. Based on our data, it seems that PLA followed by PP and PET, are more preferable for obese patients and that all tested materials can be more or less equivalently be used for lean for lean patients, assuming that a pre-dominant anti-inflammatory reaction is preferred. Although the choice of material may be better guided by the inflammatory reaction at the individual patient level rather than at the comorbidity category such as obesity. As shown in previous clinical studies, no enormous undesirable behavior of multifilament PET mesh (e.g. Parietex ${ }^{\mathrm{TM}}$ Composite mesh) for hernia repair in obese patients has been reported till now, therefore the clinical impact might be moderate[27, 28]. Nevertheless the patients outcome can always be improved with careful and personalized selection of meshes.

The polymers used in this study are commonly used for materials for soft tissue repair. The host-response to materials is not only material dependent but also the porosity, topography, and surface of the material influence the biocompatibility[3, 29-31]. The many different properties of the material influence the polarization of the macrophages[3]. In the current study, the materials were braided in the same way, but because of different diameters of the individual fibers between the materials, the topography was not exactly the same. Therefore the length of the knitted yarn was adjusted to the diameter to achieve the best possible comparable material appearance. Interestingly, 
PET and mPET resulted in different M1/M2 indexes, especially when macrophages of obese patients were cultured on the materials. This demonstrates that indeed not only the polymer but also the architecture of the material is important for elicited responses. In this study, PP did not elicit an antiinflammatory effect based on the cytokines measured. This underlines again that not only the polymer itself is important for the reaction the material elicits, but also the architecture of the material since in our earlier studies we have used meshes instead of yarns[18, 19]. Braided yarns were chosen in the current study to make the macrophage-biomaterial contact more optimal necessary for the low numbers of patient cells available for this study. After spiking of IL-6, TNFa, CCL18, and IL-1ra in the culture medium, adsorption was seen, and varying between the materials. Since PP had the most adsorption of our proteins of interest, the values for PP (and for PLA in the case of IL6) are most likely an underestimation. However, most of the associations and comparisons are made within a biomaterial and a cytokine, not comparing two different cytokines or materials with each other. These comparisons and associations are therefore unaffected in our opinion by the adsorption of the protein of interest. However, the difference in adsorption to each material, and especially the high adsorption to PP, might overshadow the differences in reactions elicited by the materials.

After implantation, the biomaterial eventually will be in contact with macrophages, but it will also be surrounded by non-adherent macrophages and extracellular matrix. We however specifically chose not to included non-adherent macrophages in our experimental set-up. The biomaterials were cultured plates made of tissue culture polystyrene (TCPS), also a polymer. By transferring the materials with their adherent cells to new wells, the medium contained mainly the cytokines from the macrophages adhering to the yarns. TCPS most likely will have a totally different effect than the extracellular matrix that normally surrounds an implanted biomaterial. In fact, we have seen that collagen indeed exerts different effect on macrophages than polymers [18, 19]. Therefore, we believed that including cytokine production from macrophages adhering to the TCPS would make the system even more artificial.

The proteins IL-6, TNF $\alpha$, CCL18, and IL-1ra were selected as indicators of pro-inflammatory and anti-inflammatory responses. We are aware that these cytokines do not represent the full spectrum of mediators produced during the foreign body response. However previously, we have seen that these mediators are most discriminative between pro-inflammatory and anti-inflammatory macrophages[18, 25]. Studies to determine the actual in vivo response to the biomaterial and correlating this with the parameters in the peripheral blood are necessary to draw more firm conclusions about the predictive value of monocyte subset percentages and serum CRP levels for the reaction biomaterials elicit in a certain patient. 


\section{Conclusion}

Monocyte-derived macrophages of obese patients respond more pro-inflammatory and less antiinflammatory to biomaterials than macrophages from lean subjects and this response depends on the type of biomaterial. This variation in cytokine production by the macrophages was associated by the percentages of monocyte subsets in the peripheral blood, serum CRP levels, or BMI of the patient. The results of this in vitro study offer possibilities and could stimulate future research towards personalized medicine, eventually leading to a model that can be used to test biomaterials for tissue repair and tissue engineering using patient's own cells prior to implantation of a biomaterial. In addition, our results offer the prospect that monocyte subsets in the blood or serum CRP might be measured prior to surgery to predict which biomaterial might be suitable for each patient. Studies indeed examining the clinical outcome after implantation of a biomaterial in relation to serum CRP, BMI, and monocyte subsets are however needed to confirm this.

\section{Acknowledgements}

We would like to thank all volunteers and patients who participated in this study. This study is supported by Medtronic-Sofradim, but they were not involved in the design, conduct or data analysis of the study. Yvonne Bastiaansen-Jenniskens was supported by a Veni grant from NOW/STW. The authors declare no conflict of interest. 
Figure 1 Experimental flow of our study, including pictures of the yarns and how the yarns were placed in the culture wells. CD14 = cluster of differentiation $14, \mathrm{PP}=$ polypropylene, $\mathrm{PLA}=$ polylactic acid, $\mathrm{PET}=$ monofilament polyethylene terephthalate, and $\mathrm{mPET}=$ multifilament polyethylene terephthalate.

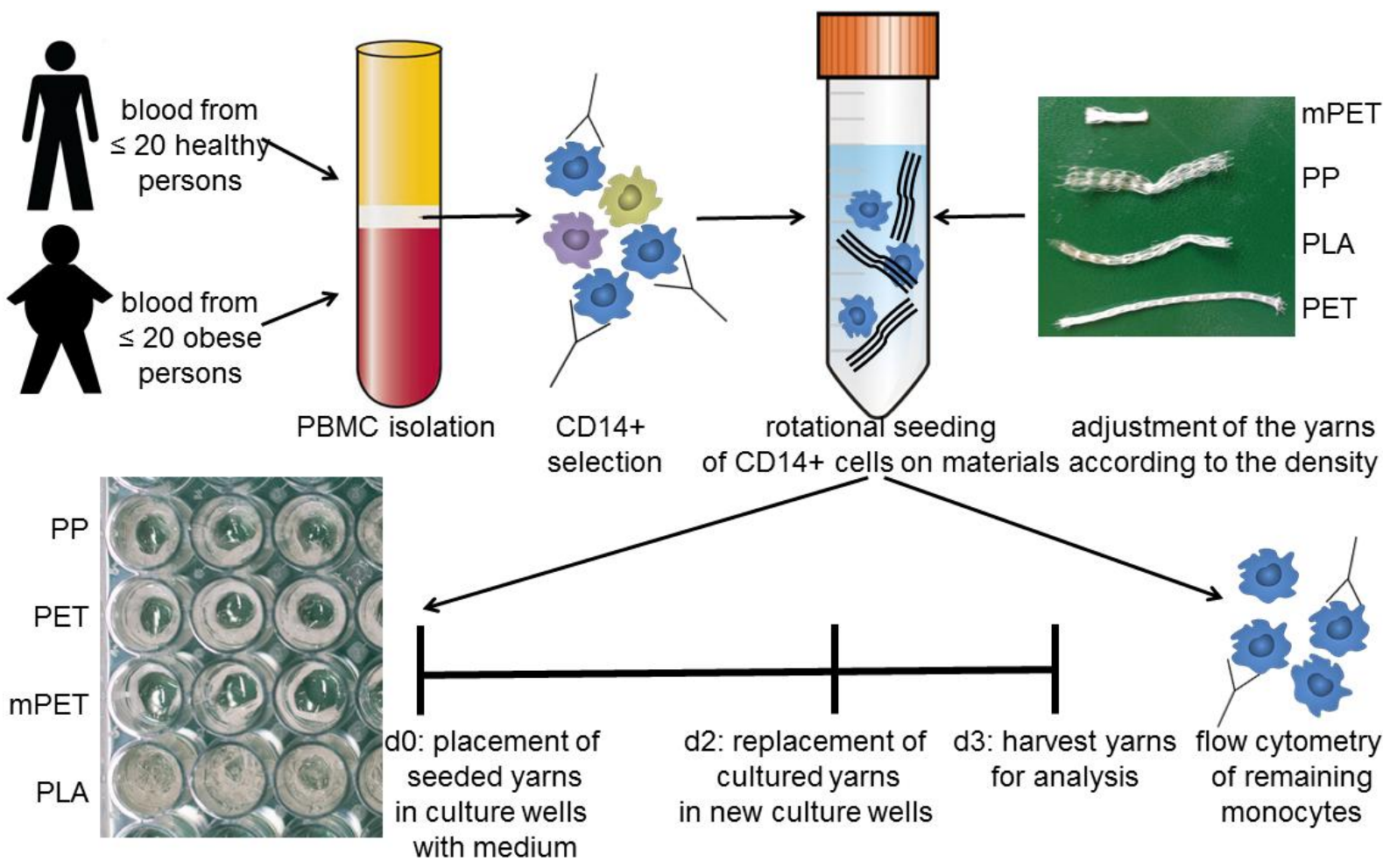


Table 1. Patient characteristics lean group vs obese patients.

\begin{tabular}{|c|c|c|c|}
\hline & lean $(n=20)$ & obese $(n=20)$ & $p$-value \\
\hline $\mathrm{BMI}\left(\mathrm{kg} / \mathrm{m}^{2}\right)$ & $22.9 \pm 2.6$ & $43.8 \pm 6.5$ & $<0.001$ \\
\hline gender $($ male/ female) & $2 / 18$ & $2 / 18$ & 1.0 \\
\hline age (years) & $41.8 \pm 13.1$ & $41.3 \pm 13.5$ & 0.916 \\
\hline use of statins & $0 / 20$ & $2 / 20$ & 0.154 \\
\hline
\end{tabular}

Values are means (SD), p-value was estimated by using independent sample T-test

Table 2. The average production of IL-6, TNF $\alpha$, CCL18, and IL-1ra corrected for DNA by macrophages on the different materials.

\begin{tabular}{|c|c|c|c|c|}
\hline \multirow[b]{2}{*}{ cytokine } & \multicolumn{4}{|c|}{$\begin{array}{l}\text { cytokine production by macrophages } \\
\text { (pg protein/ng DNA) }\end{array}$} \\
\hline & material & $\begin{array}{c}\text { lean } \\
(\text { mean } \pm S D)\end{array}$ & $\begin{array}{c}\text { obese } \\
\text { (mean } \pm \text { SD) }\end{array}$ & $p$ value \\
\hline \multirow[t]{4}{*}{ IL-6 } & PP & $116.6 \pm 97.2$ & $172.4 \pm 114.1$ & 0.106 \\
\hline & PLA & $109.2 \pm 67.1$ & $157.4 \pm 146.8$ & 0.247 \\
\hline & PET & $102.0 \pm 73.9$ & $144.0 \pm 58.4$ & 0.022 \\
\hline & mPET & $39.2 \pm 23.9$ & $68.7 \pm 64.0$ & 0.140 \\
\hline \multirow[t]{4}{*}{ TNF $\alpha$} & PP & $7.9 \pm 8.3$ & $5.9 \pm 4.9$ & 0.300 \\
\hline & PLA & $5.0 \pm 4.4$ & $3.3 \pm 3.1$ & 0.119 \\
\hline & PET & $3.6 \pm 3.8$ & $3.3 \pm 1.5$ & 0.417 \\
\hline & mPET & $1.0 \pm 0.9$ & $0.7 \pm 0.5$ & 0.421 \\
\hline \multirow[t]{4}{*}{ CCL18 } & PP & $0.8 \pm 0.7$ & $0.2 \pm 0.3$ & $<0.001$ \\
\hline & PLA & $1.4 \pm 0.9$ & $0.4 \pm 0.4$ & 0.002 \\
\hline & PET & $1.6 \pm 1.3$ & $0.5 \pm 0.6$ & 0.002 \\
\hline & mPET & $0.7 \pm 0.6$ & $0.3 \pm 0.3$ & 0.007 \\
\hline \multirow[t]{4}{*}{ IL-1ra } & PP & $49.4 \pm 52.2$ & $24.4 \pm 16.8$ & 0.128 \\
\hline & PLA & $34.6 \pm 28.5$ & $15.5 \pm 9.0$ & 0.026 \\
\hline & PET & $32.3 \pm 22.6$ & $20.2 \pm 13.6$ & 0.071 \\
\hline & mPET & $18.0 \pm 18.4$ & $10.0 \pm 12.3$ & 0.057 \\
\hline
\end{tabular}

Bold values denote statistical significance 
Figure 2: Measurements of IL-6 (A), TNF $\alpha$ (B), CCL18 (C), and IL-1ra (D) in the culture medium with and without the incubation of the biomaterials and with and without spiking of the protein of interest. White bar indicates measurements in medium with or without incubation of the materials. Black bars indicate measurements in medium alone or after incubation with the material in the presence of the spiked proteins. Bars represent $n=6+s d$ for every bar.

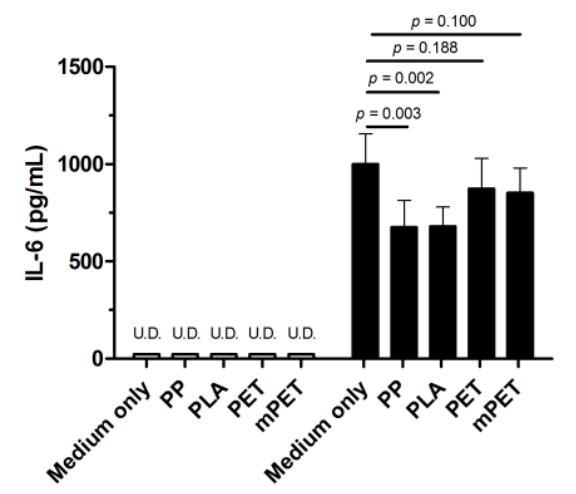

$$
\begin{aligned}
& \square \text { - IL-6 } \\
& \text { + IL-6 } \\
& \text { Detection limit: } 23.44 \text { pg/mL }
\end{aligned}
$$

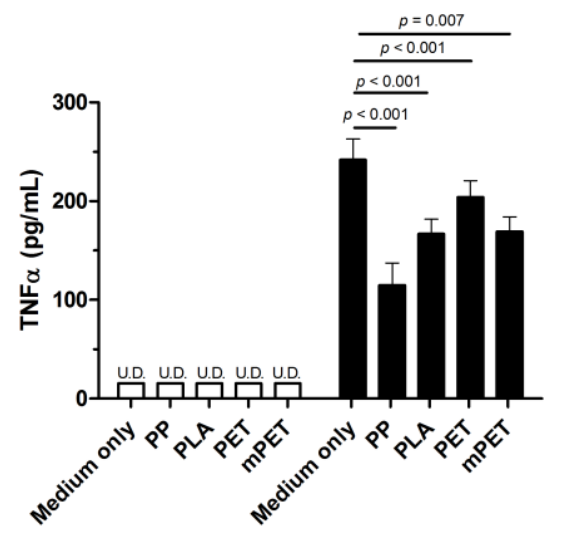

$$
\begin{aligned}
& \square-\mathrm{TNF} \alpha \\
& \text { + TNF } \alpha \\
& \text { Detection limit: } 15.6 \mathrm{pg} / \mathrm{mL}
\end{aligned}
$$
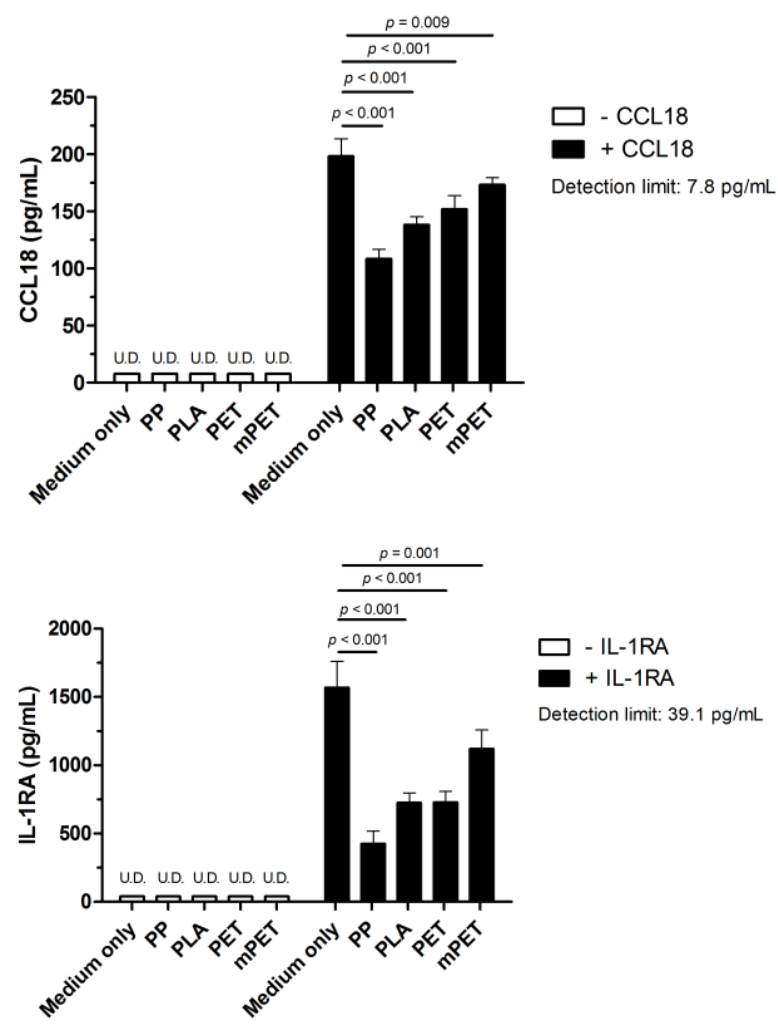
Figure 3 Cytokine production corrected for DNA compared per material, in lean subjects or in obese subjects. Number of patients per cytokine and per material are indicated in the bars or just above the error bar.
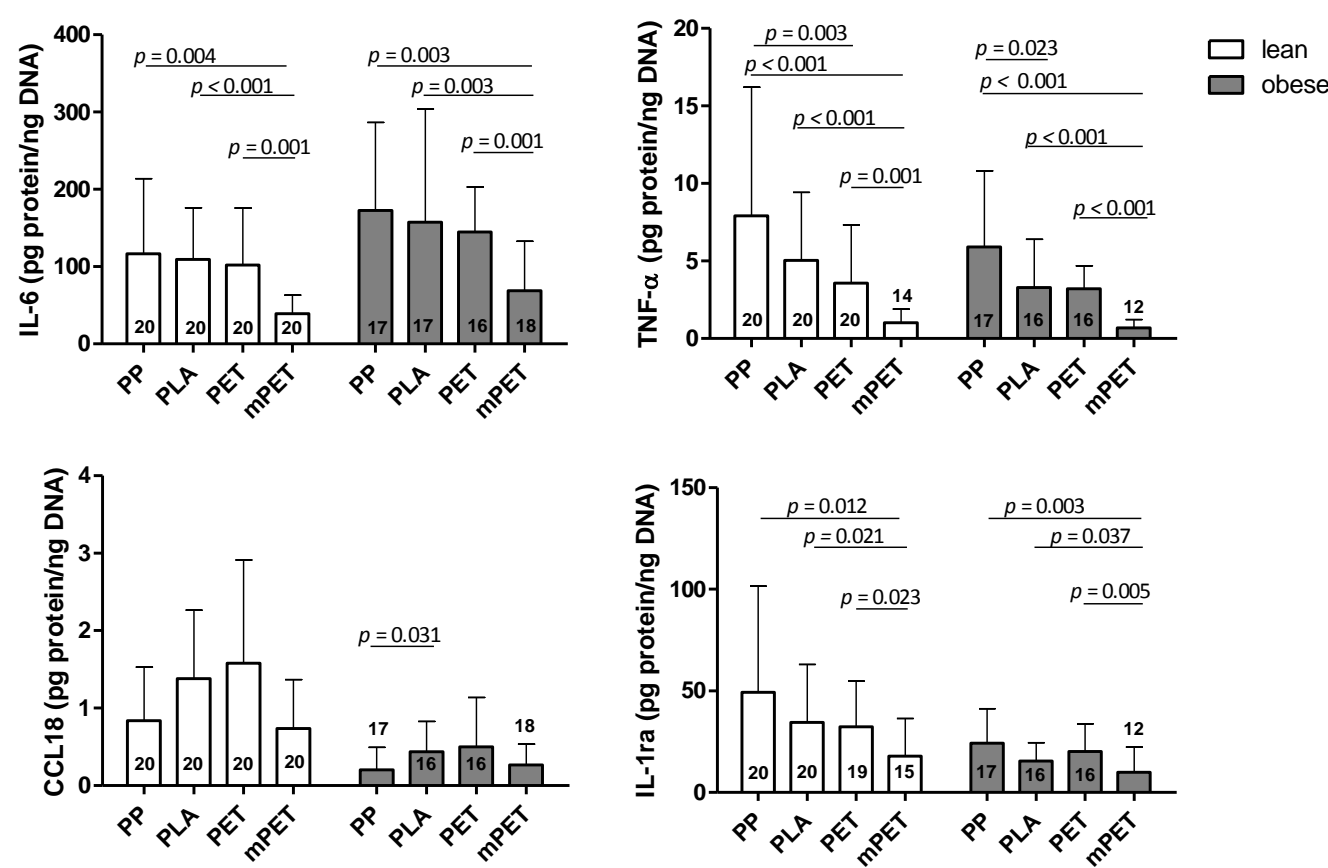
Figure 4: $M 1 / M 2$ index between materials. Bars represent the mean, error bars the $S D, p$-values indicate significant differences. A base 2-log scale is used for the $\mathrm{Y}$-axis. PP = polypropylene, PLA = polylactic acid, PET = monofilament polyethylene terephthalate, and $\mathrm{mPET}=$ multifilament polyethylene terephthalate. The number of patients included per material, per group are indicated in the bars.

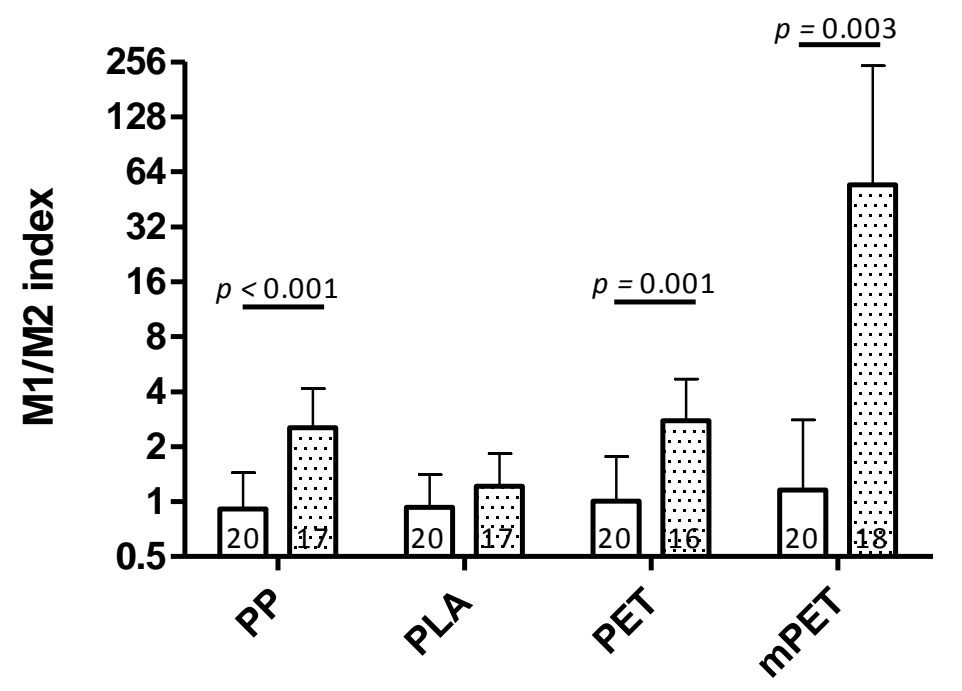


Table 3 Correlations between CRP concentration in peripheral blood, BMI of all subjects, and cytokine production by the macrophages.

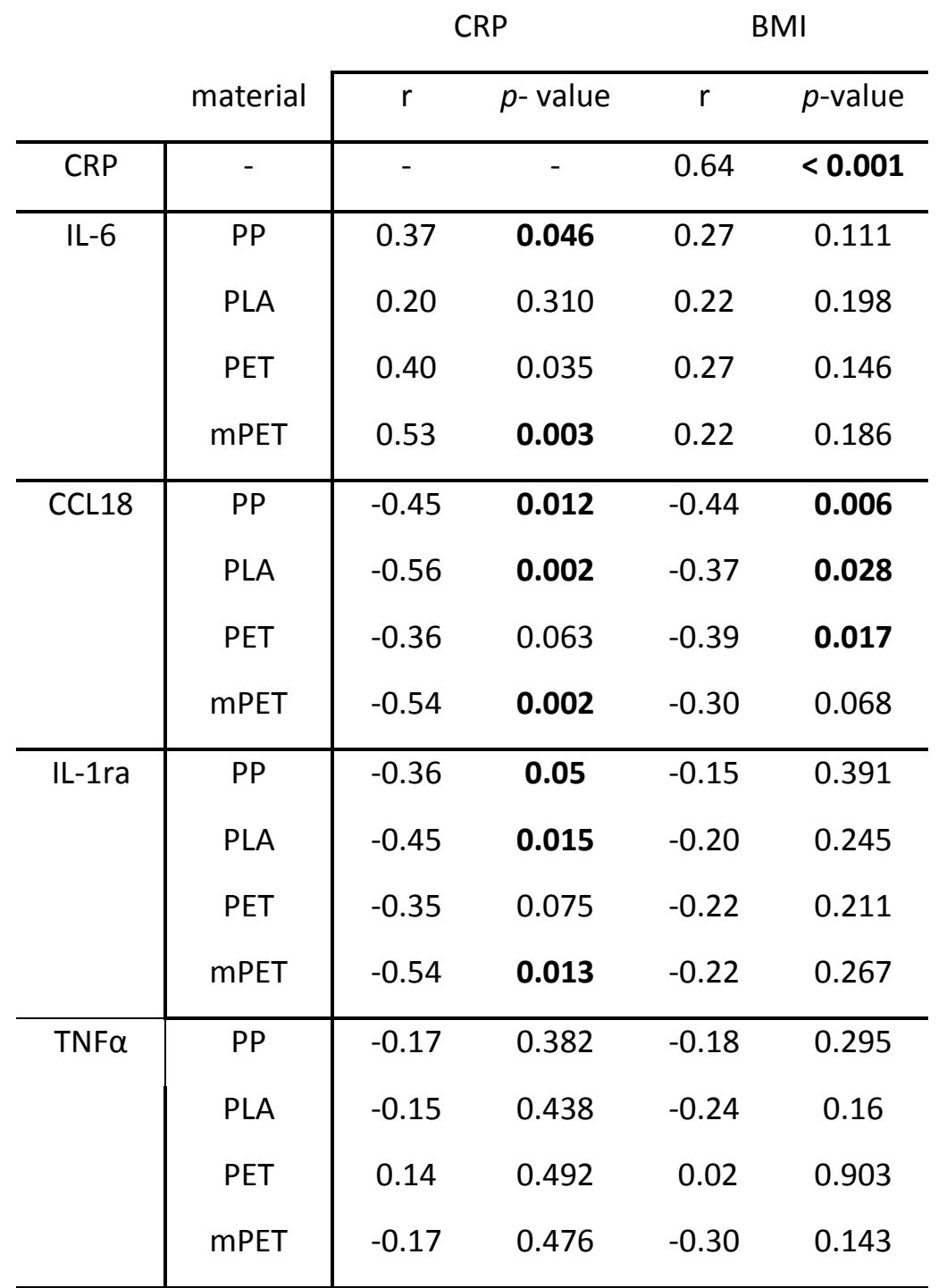

Bold values denote statistically significant $p$-values

Table 4 Percentages of peripheral blood monocytes subsets in lean $(n=9)$ and obese $(n=8)$ subjects. Values are mean $+/-$ sd.

\begin{tabular}{c|ccc} 
\% of monocyte & lean & obese & $p$-value \\
\hline classical $\left(\mathrm{CD} 14^{++} \mathrm{CD} 16\right)$ & $90.9 \pm 5.3$ & $77.4 \pm 22.0$ & 0.290 \\
intermediate $\left(\mathrm{CD} 14^{++} \mathrm{CD} 16^{+}\right)$ & $2.2 \pm 3.4$ & $7.9 \pm 13.4$ & 0.336 \\
non-classical $\left(\mathrm{CD} 14^{+} \mathrm{CD} 16^{++}\right)$ & $4.0 \pm 3.8$ & $12.9 \pm 13.7$ & 0.211
\end{tabular}


Table 5A Spearman correlation between percentages of $\mathrm{CD} 14^{++} \mathrm{CD} 16^{-}$(classical), $\mathrm{CD} 14^{++} \mathrm{CD} 16^{+}$ (intermediate), or $\mathrm{CD} 14^{+} \mathrm{CD} 16^{++}$(non-classical) monocyte subsets and production of cytokines by cultured macrophages on the four different materials. The Spearman correlation coefficients $(r)$ define the relationship between monocyte subsets from peripheral blood and the production of IL-6, CCL18, IL-1ra, and TNF $\alpha$ ) by macrophages cultured on PP, PLA, PET, and MPET. Table 5B shows the correlation between the percentages of monocyte subsets with the $M 1 / M 2$ index for the four different materials. $\mathrm{PP}=$ polypropylene, $\mathrm{PLA}=$ polylactic acid, $\mathrm{PET}=$ monofilament polyethylene terephthalate, and $\mathrm{mPET}=$ multifilament polyethylene terephthalate.

\begin{tabular}{|c|c|c|c|c|c|c|c|}
\hline \multirow[t]{2}{*}{ A } & \multirow[b]{2}{*}{ material } & \multicolumn{2}{|c|}{$\mathrm{CD} 14^{++} \mathrm{CD} 16^{-}$} & \multicolumn{2}{|c|}{$\mathrm{CD} 14^{++} \mathrm{CD} 16^{+}$} & \multicolumn{2}{|c|}{$\mathrm{CD} 14^{+} \mathrm{CD} 16^{++}$} \\
\hline & & $r$ & $p$-value & $r$ & $p$-value & $r$ & $p$-value \\
\hline CRP & - & -0.42 & 0.120 & 0.35 & 0.198 & 0.35 & 0.203 \\
\hline $\mathrm{BMI}$ & & -0.16 & 0.535 & 0.12 & 0.636 & 0.31 & 0.231 \\
\hline \multirow[t]{4}{*}{ IL-6 } & PP & -0.17 & 0.541 & 0.27 & 0.334 & 0.26 & 0.355 \\
\hline & PLA & -0.43 & 0.086 & 0.53 & 0.028 & 0.41 & 0.103 \\
\hline & PET & -0.54 & 0.038 & 0.51 & 0.052 & 0.47 & 0.079 \\
\hline & mPET & -0.36 & 0.158 & 0.38 & 0.133 & 0.26 & 0.323 \\
\hline \multirow[t]{4}{*}{ CCL18 } & PP & 0.28 & 0.321 & -0.13 & 0.639 & -0.23 & 0.405 \\
\hline & PLA & 0.16 & 0.549 & -0.32 & 0.107 & -0.18 & 0.370 \\
\hline & PET & 0.21 & 0.451 & -0.40 & 0.045 & -0.36 & 0.073 \\
\hline & mPET & 0.24 & 0.353 & -0.50 & 0.007 & -0.39 & 0.039 \\
\hline \multirow[t]{4}{*}{ IL-1ra } & $\mathrm{PP}$ & 0.12 & 0.676 & -0.43 & 0.108 & 0.18 & 0.516 \\
\hline & PLA & 0.16 & 0.529 & -0.29 & 0.252 & 0.04 & 0.889 \\
\hline & PET & 0.28 & 0.334 & -0.65 & 0.011 & -0.03 & 0.911 \\
\hline & mPET & 0.13 & 0.658 & -0.53 & 0.052 & 0.16 & 0.594 \\
\hline \multirow[t]{4}{*}{ TNF $\alpha$} & PP & -0.37 & 0.173 & 0.17 & 0.550 & 0.42 & 0.121 \\
\hline & PLA & -0.35 & 0.171 & 0.37 & 0.141 & 0.21 & 0.428 \\
\hline & PET & -0.46 & 0.084 & 0.30 & 0.283 & 0.30 & 0.296 \\
\hline & mPET & -0.41 & 0.167 & 0.01 & 0.986 & 0.42 & 0.152 \\
\hline
\end{tabular}

B Spearman correlation between percentages of monocyte subsets and M1/M2 index

\begin{tabular}{|c|c|c|c|c|c|c|c|}
\hline & \multirow[b]{2}{*}{ material } & \multicolumn{2}{|c|}{$\mathrm{CD}_{14} 4^{++} \mathrm{CD} 16^{-}$} & \multicolumn{2}{|c|}{$\mathrm{CD} 14^{++} \mathrm{CD} 16^{+}$} & \multicolumn{2}{|c|}{$\mathrm{CD} 14^{+} \mathrm{CD} 16^{++}$} \\
\hline & & $r$ & $p$-value & $\mathrm{R}$ & p-value & $r$ & $p$-value \\
\hline \multirow[t]{4}{*}{$\mathrm{M} 1 / \mathrm{M} 2$} & PP & -0.59 & 0.020 & 0.36 & 0.182 & 0.48 & 0.069 \\
\hline & PLA & -0.40 & 0.112 & 0.37 & 0.144 & 0.35 & 0.174 \\
\hline & PET & -0.45 & 0.092 & 0.26 & 0.341 & 0.27 & 0.328 \\
\hline & mPET & -0.51 & 0.038 & 0.58 & 0.016 & 0.41 & 0.098 \\
\hline
\end{tabular}

Bold values denote statistically significant $p$-values 


\section{References}

1. Binyamin, G., B.M. Shafi, and C.M. Mery, Biomaterials: a primer for surgeons. Semin Pediatr Surg, 2006. 15(4): p. 276-83.

2. Anderson, J.M., A. Rodriguez, and D.T. Chang, Foreign body reaction to biomaterials. Semin Immunol, 2008. 20(2): p. 86-100.

3. Sridharan, R., et al., Biomaterial based modulation of macrophage polarization: a review and suggested design principles. Materials Today, 2015. 18(6): p. 313-325.

4. Pierpont, Y.N., et al., Obesity and surgical wound healing: a current review. ISRN Obes, 2014. 2014: p. 638936.

5. Rosemar, A., et al., Effect of body mass index on groin hernia surgery. Ann Surg, 2010. 252(2): p. 397-401.

6. Komen, N., et al., After-hours colorectal surgery: a risk factor for anastomotic leakage. Int J Colorectal Dis, 2009. 24(7): p. 789-95.

7. Sauerland, S., et al., Obesity is a risk factor for recurrence after incisional hernia repair. Hernia, 2004. 8(1): p. 42-6.

8. Langer, S. and J. Christiansen, Long-term results after incisional hernia repair. Acta Chir Scand, 1985. 151(3): p. 217-9.

9. Lumeng, C.N., J.L. Bodzin, and A.R. Saltiel, Obesity induces a phenotypic switch in adipose tissue macrophage polarization. J Clin Invest, 2007. 117(1): p. 175-84.

10. Lumeng, C.N., et al., Phenotypic switching of adipose tissue macrophages with obesity is generated by spatiotemporal differences in macrophage subtypes. Diabetes, 2008. 57(12): p. 3239-46.

11. Considine, R.V., Activated monocytes: yet another link between systemic inflammation and obesity. J Clin Endocrinol Metab, 2014. 99(7): p. 2347-9.

12. $\mathrm{Xu}, \mathrm{H}$., et al., Chronic inflammation in fat plays a crucial role in the development of obesityrelated insulin resistance. J Clin Invest, 2003. 112(12): p. 1821-30.

13. Kammoun, H.L., M.J. Kraakman, and M.A. Febbraio, Adipose tissue inflammation in glucose metabolism. Rev Endocr Metab Disord, 2014. 15(1): p. 31-44.

14. Ziegler-Heitbrock, L., Monocyte subsets in man and other species. Cell Immunol, 2014. 289(12): p. 135-9.

15. Martinez, F.O., The transcriptome of human monocyte subsets begins to emerge. J Biol, 2009. 8(11): p. 99.

16. Rogacev, K.S., et al., Monocyte heterogeneity in obesity and subclinical atherosclerosis. Eur Heart J, 2010. 31(3): p. 369-76.

17. Poitou, C., et al., CD14dimCD16+ and CD14+CD16+ monocytes in obesity and during weight loss: relationships with fat mass and subclinical atherosclerosis. Arterioscler Thromb Vasc Biol, 2011. 31(10): p. 2322-30.

18. Grotenhuis, N., et al., A culture model to analyze the acute biomaterial-dependent reaction of human primary macrophages. Biochem Biophys Res Commun, 2013. 433(1): p. 115-20.

19. Grotenhuis, N., et al., In vitro model to study the biomaterial-dependent reaction of macrophages in an inflammatory environment. Br J Surg, 2014. 101(8): p. 983-92.

20. Utomo, L., et al., Guiding synovial inflammation by macrophage phenotype modulation: an in vitro study towards a therapy for osteoarthritis. Osteoarthritis Cartilage, 2016.

21. Grundy, S.M., et al., Definition of metabolic syndrome: Report of the National Heart, Lung, and Blood Institute/American Heart Association conference on scientific issues related to definition. Circulation, 2004. 109(3): p. 433-8.

22. Devaraj, S. and I. Jialal, C-reactive protein polarizes human macrophages to an M1 phenotype and inhibits transformation to the M2 phenotype. Arterioscler Thromb Vasc Biol, 2011. 31(6): p. 1397-402.

23. Krinninger, P., et al., Peripheral monocytes of obese women display increased chemokine receptor expression and migration capacity. J Clin Endocrinol Metab, 2014. 99(7): p. 2500-9. 
24. Cottam, D.R., et al., Effect of surgically-induced weight loss on leukocyte indicators of chronic

25. Lopa, S., et al., Arthritic and non-arthritic synovial fluids modulate IL10 and IL1RA gene expression in differentially activated primary human monocytes. Osteoarthritis Cartilage, 2015. 23(11): p. 1853-7.

26. Gratchev, A., et al., Mphi1 and Mphi2 can be re-polarized by Th2 or Th1 cytokines, respectively, and respond to exogenous danger signals. Immunobiology, 2006. 211(6-8): p. 473-86.

27. Ching, S.S., et al., Comparison of early outcomes for laparoscopic ventral hernia repair between nonobese and morbidly obese patient populations. Surg Endosc, 2008. 22(10): p. 2244-50.

28. Briennon, X., et al., Surgical treatment of large incisional hernias by intraperitoneal insertion of Parietex(R) composite mesh with an associated aponeurotic graft (280 cases). J Visc Surg, 2011. 148(1): p. 54-8.

29. Harvey, A.G., E.W. Hill, and A. Bayat, Designing implant surface topography for improved biocompatibility. Expert Rev Med Devices, 2013. 10(2): p. 257-67.

30. Roach, P., et al., Modern biomaterials: a review - bulk properties and implications of surface modifications. J Mater Sci Mater Med, 2007. 18(7): p. 1263-77.

31. Rosengren, A. and L.M. Bjursten, Pore size in implanted polypropylene filters is critical for tissue organization. J Biomed Mater Res A, 2003. 67(3): p. 918-26. 


\section{Supplementary data}

Supplementary figure 1; flow cytometric analysis of fresh (A, B), and frozen monocytes (C, D). Forward scatter (FSC) and sideward scatter (SSC) show size and granularity of the cells $(A, C)$ and monocyte subsets were determined based on the presence of cluster of differentiation 14 (CD14) and CD16 (B, D).

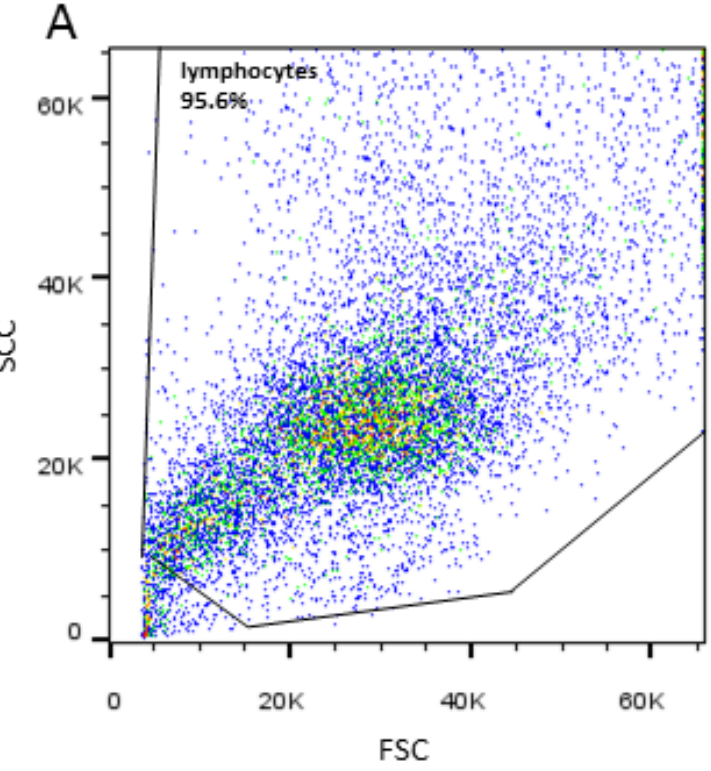

C

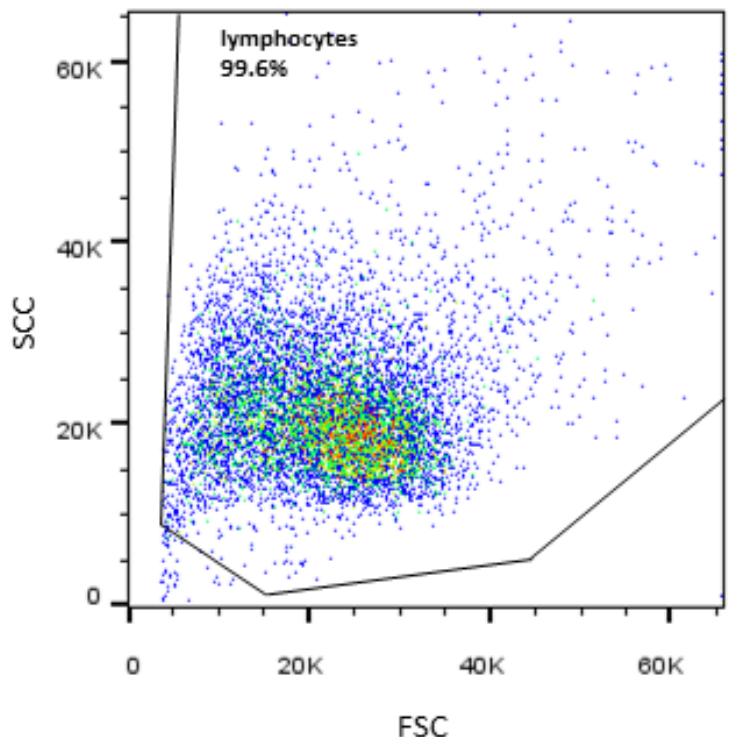

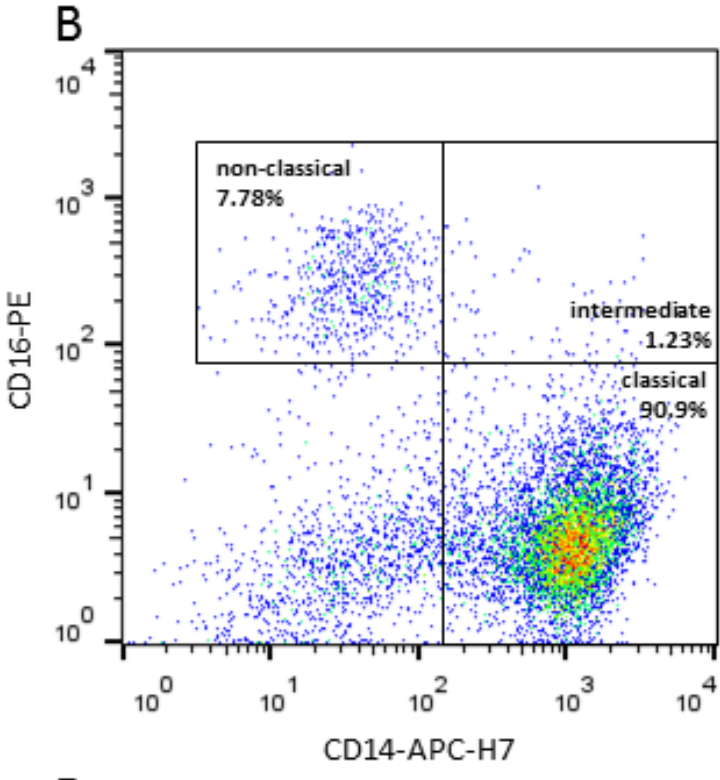

D

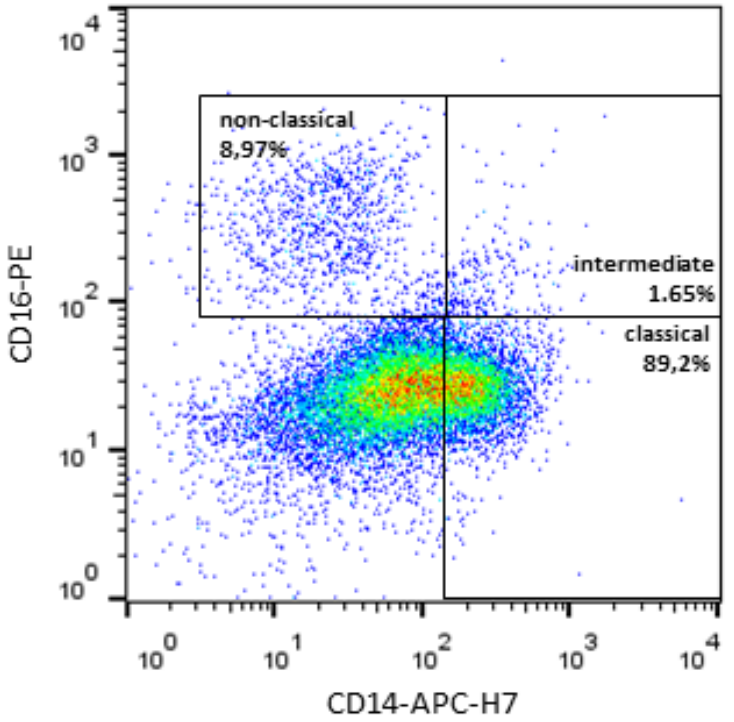


Supplementary Figure 2A: IL-6, B: TNF $\alpha$, C CCL18, D IL-1ra production by macrophages seeded on

different materials corrected for DNA, lean vs. obese groups shown per material. Every dot represents a single donor. The line indicates the mean, $p$-values indicate a statistically significant difference. Bars represent the mean, whiskers the SD. Ratios underneath the graphs indicate the male/female ratio per measurement and per material. $\mathrm{PP}=$ polypropylene, $\mathrm{PLA}=$ polylactic acid, $\mathrm{PET}$ $=$ monofilament polyethylene terephthalate, and $\mathrm{mPET}=$ multifilament polyethylene terephthalate.

\section{A}
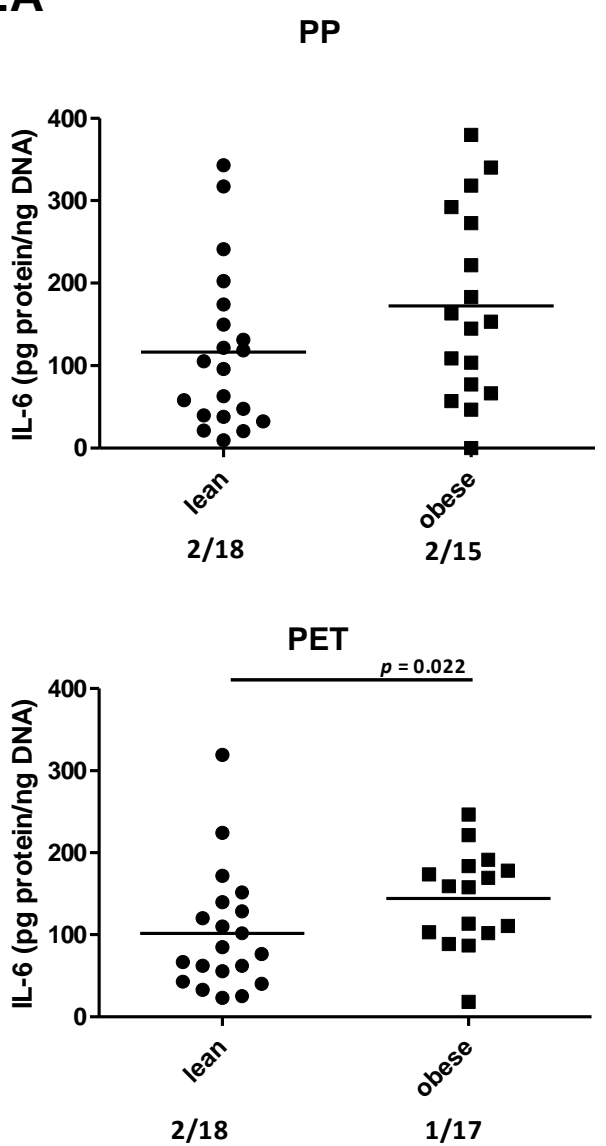

PLA

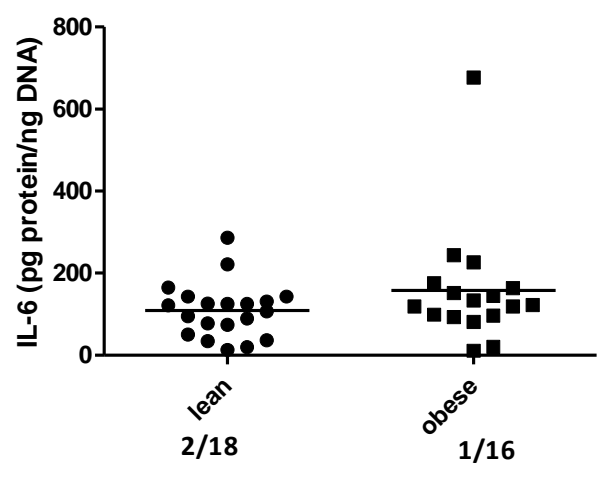

MPET

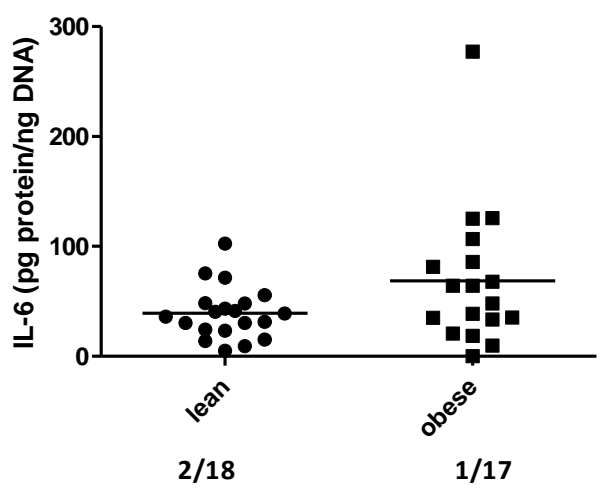


2B
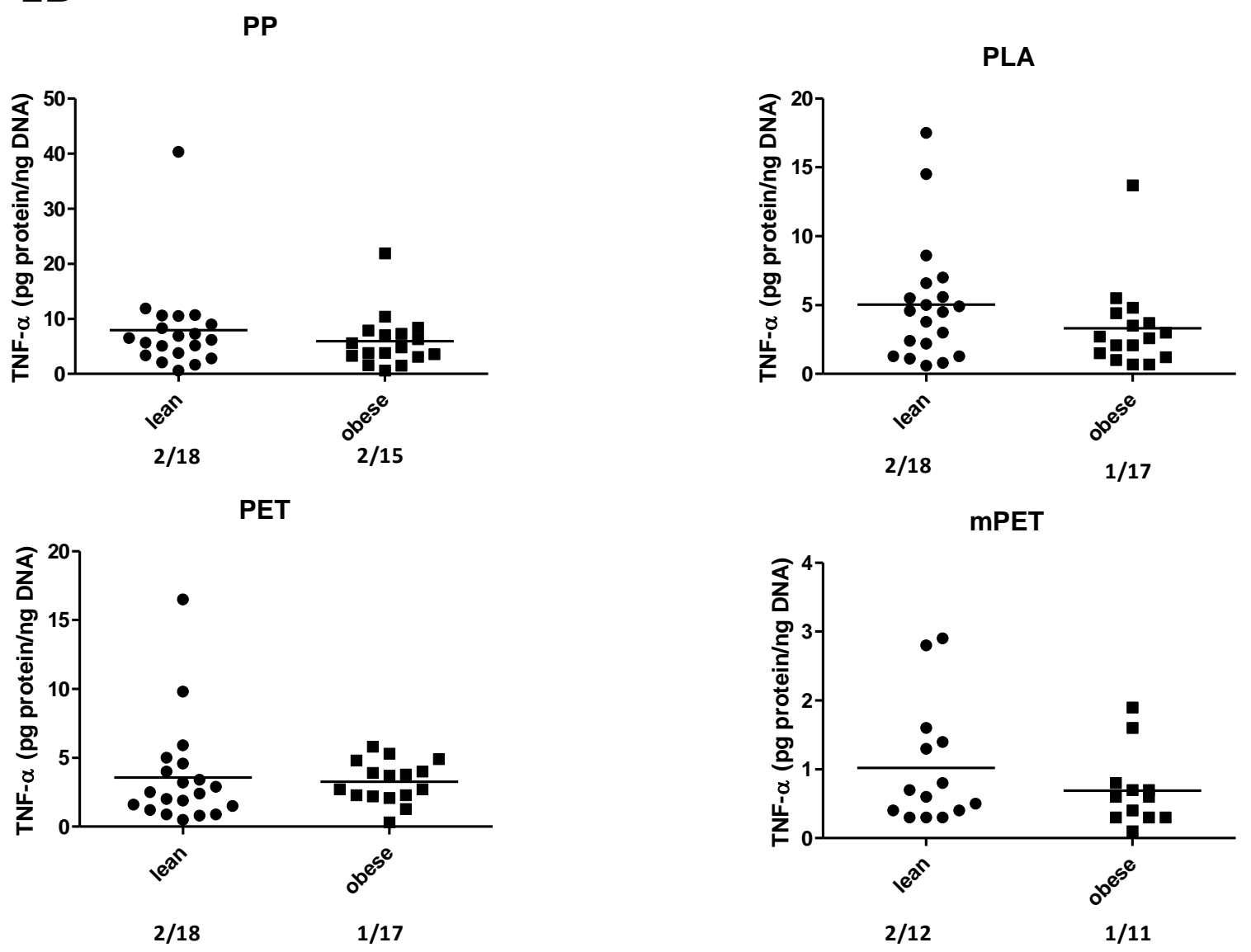
2C
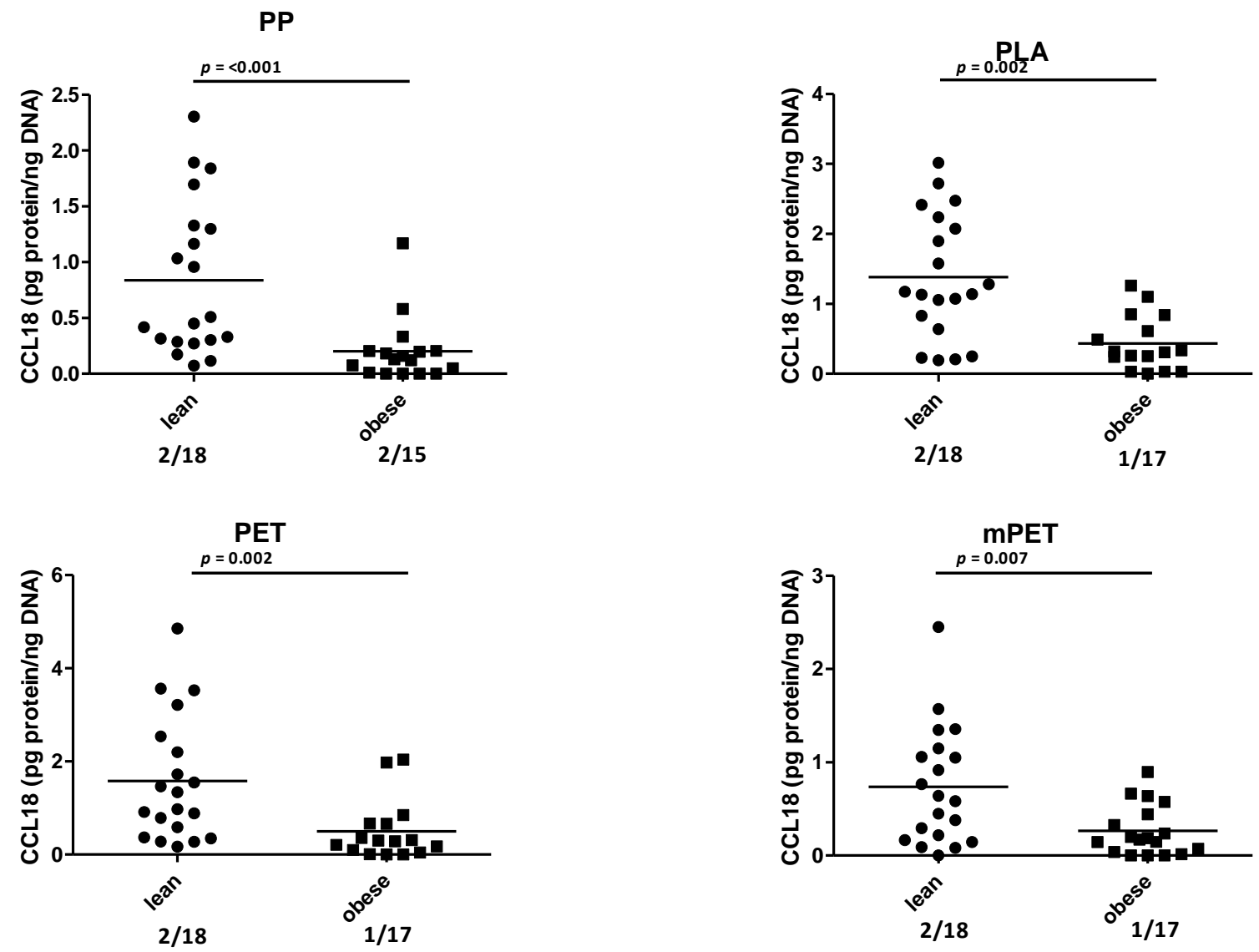

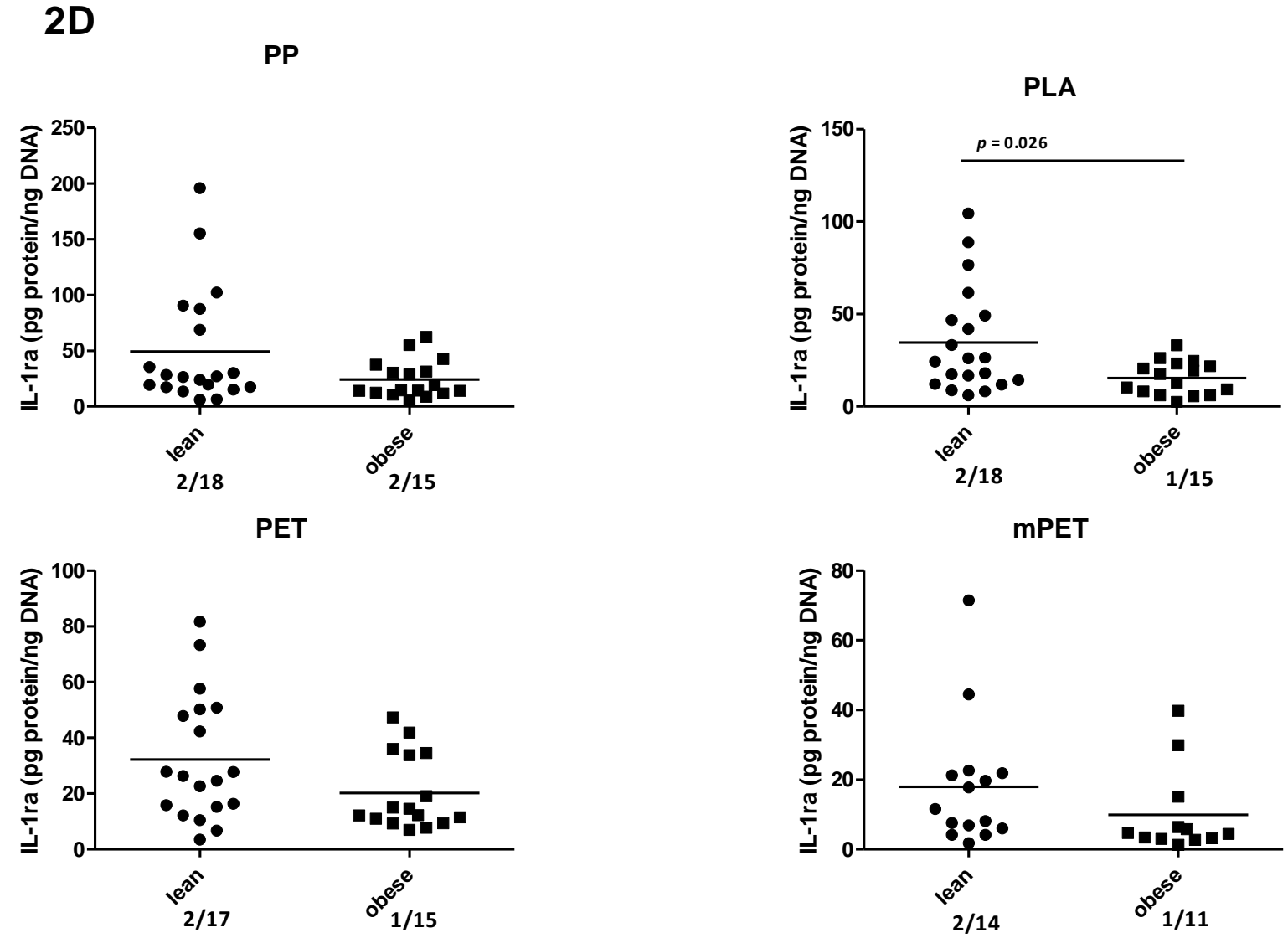
Supplementary Figure 3A. The amount of DNA as indication of the number of attached macrophages to the biomaterials. DNA is shown as $\mathrm{ng} / \mathrm{mL}$ for polypropylene (PP), polylactic acid (PLA), monofilament polyethylene terephthalate (PET), and multifilament polyethylene terephthalate (mPET) for the lean (open bars) and obese (dotted bars) donors. Bars represent mean \pm S.E.M. $n=20$ donors/group, three samples/ per material/ per donor, $p$-value indicates a significant difference. Figure 3B, C, D, E Comparing macrophages from lean and obese donors cultured on different materials regarding B) IL-6 production and C) TNF $\alpha$ production and D) CCL18 production and E) IL-1ra production in $\mathrm{ng} / \mathrm{ml}$ after 3 days of culture. Every dot represents a single donor. Line and whiskers indicate mean \pm S.E.M., p-values indicate a statistically significant difference. PP = polypropylene, PLA $=$ polylactic acid, $\mathrm{PET}=$ monofilament polyethylene terephthalate, and $\mathrm{mPET}=$ multifilament polyethylene terephthalate.

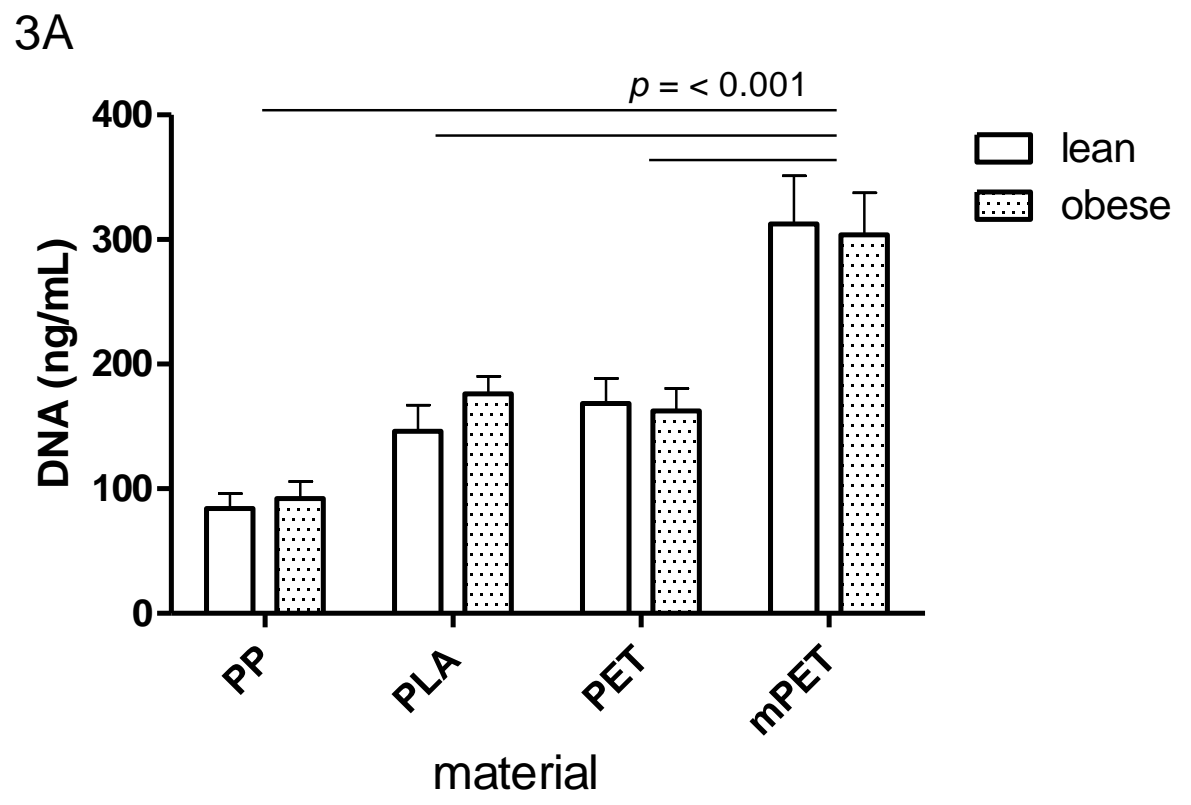


B
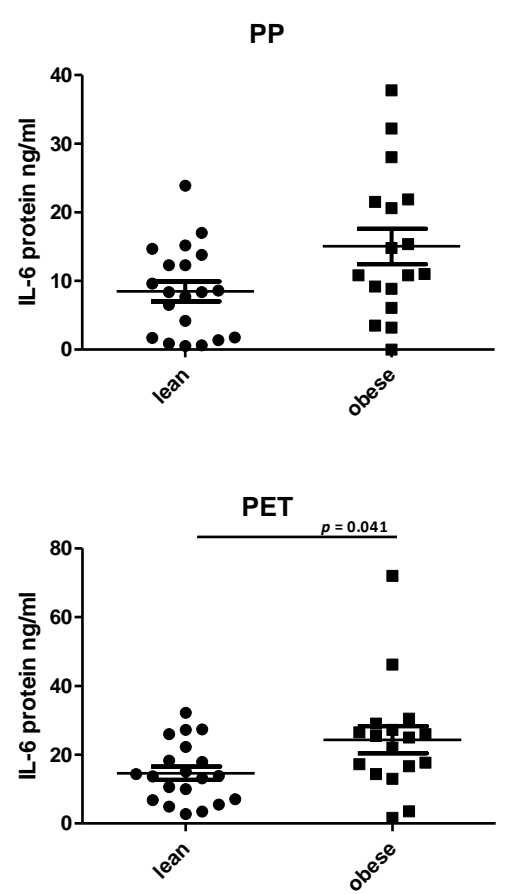

C

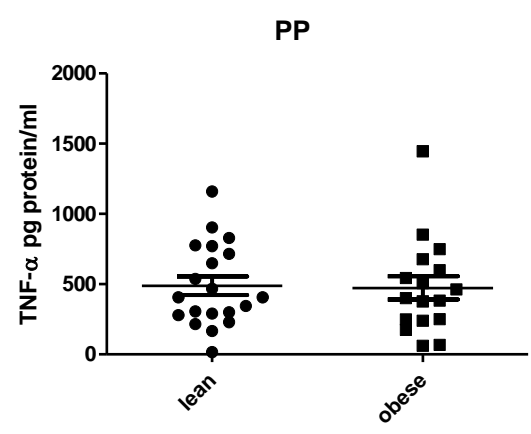

PET

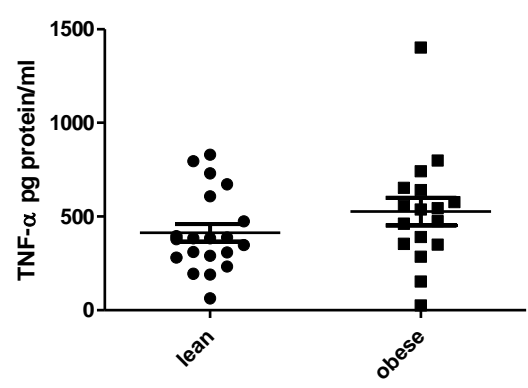

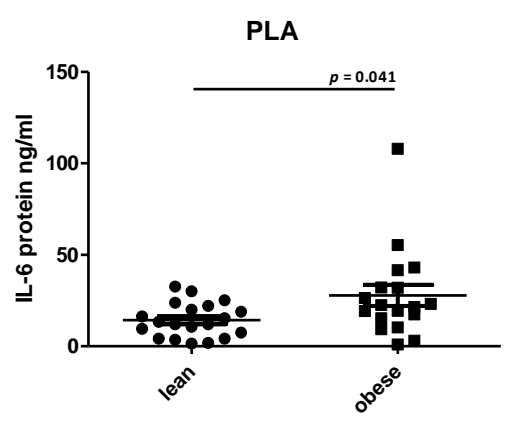

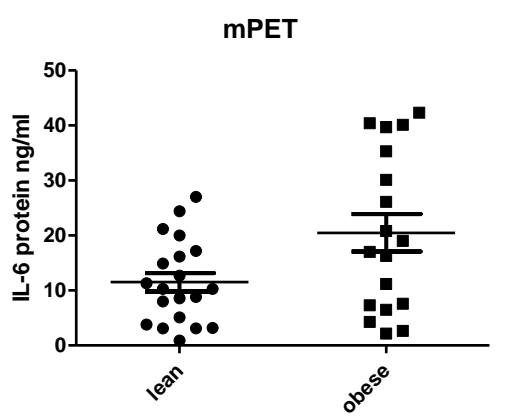

PLA
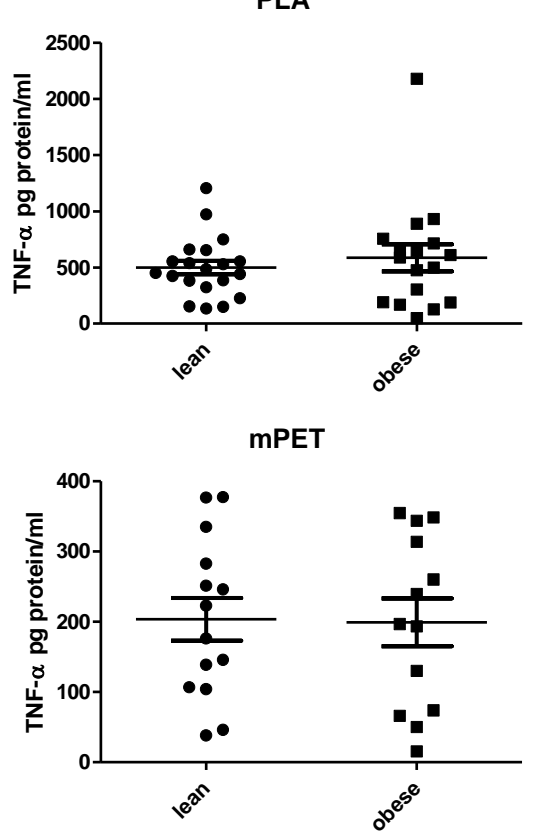
D
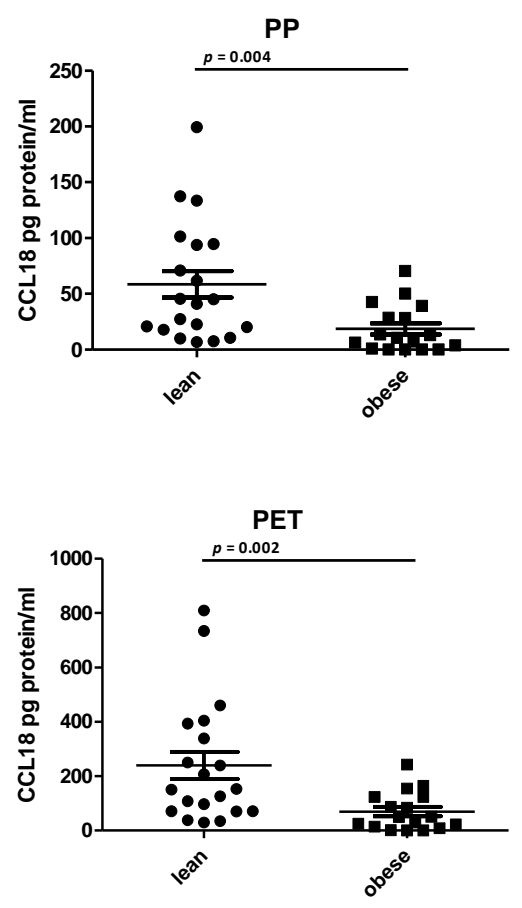

E

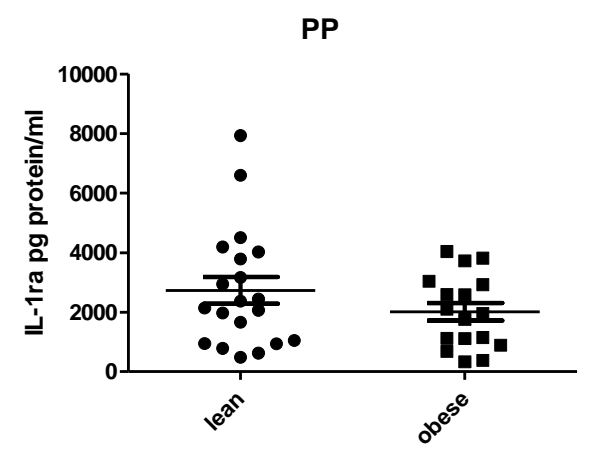

PET

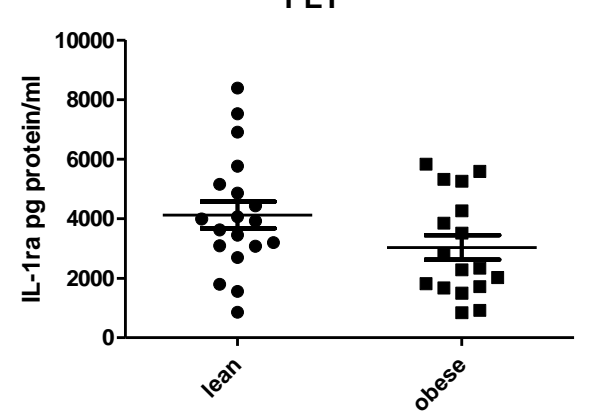

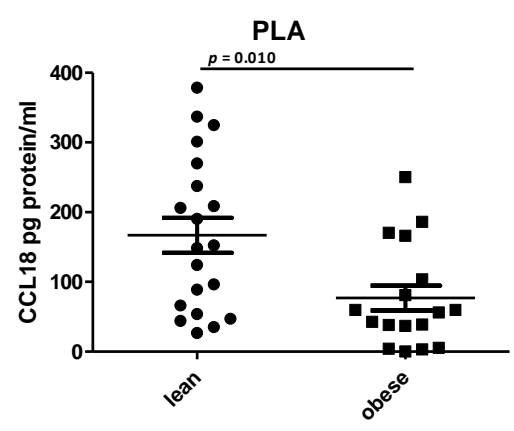
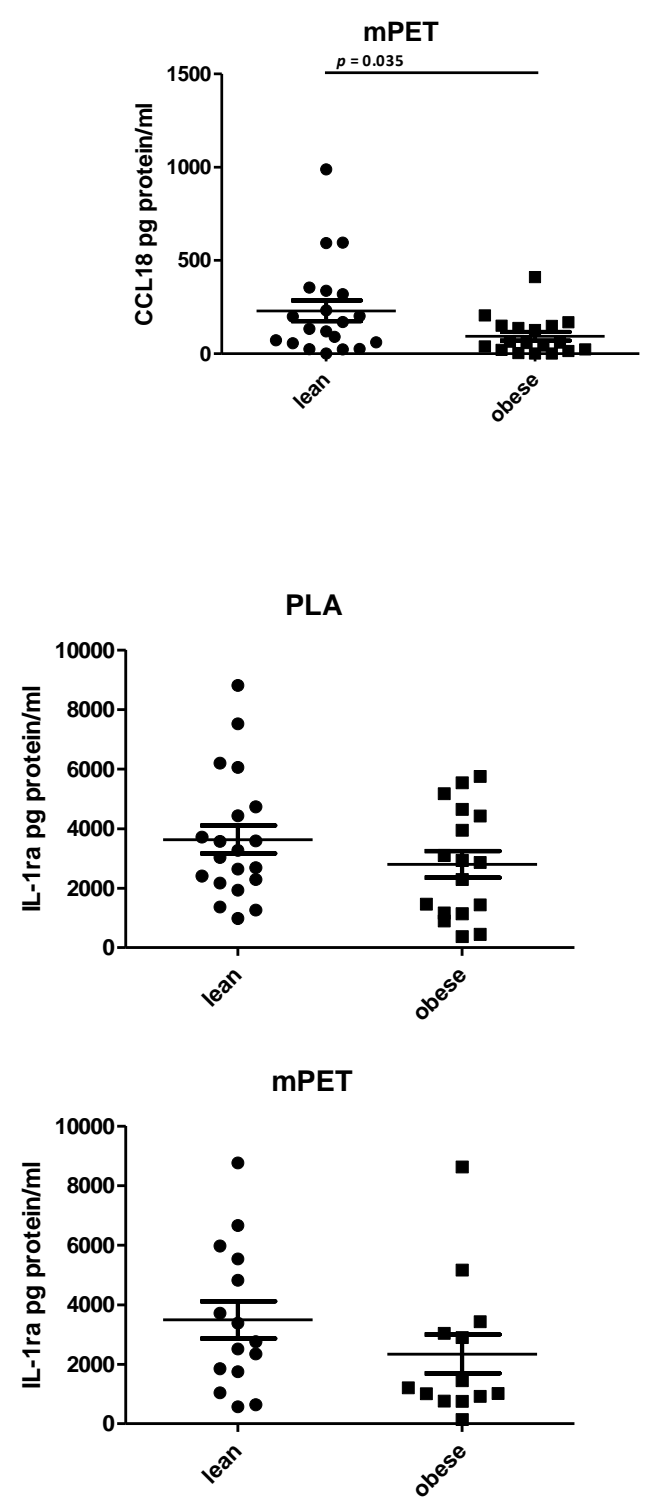
Figure 4 C-reactive protein (CRP) levels in $\mathrm{mg} / \mathrm{L}$ in plasma of lean subjects vs. obese patients. The middle line in box represent the median and whiskers the minimum and maximum; lean $(0-7 \mathrm{mg} / \mathrm{L})$ and obese $(0-75 \mathrm{mg} / \mathrm{L})$.

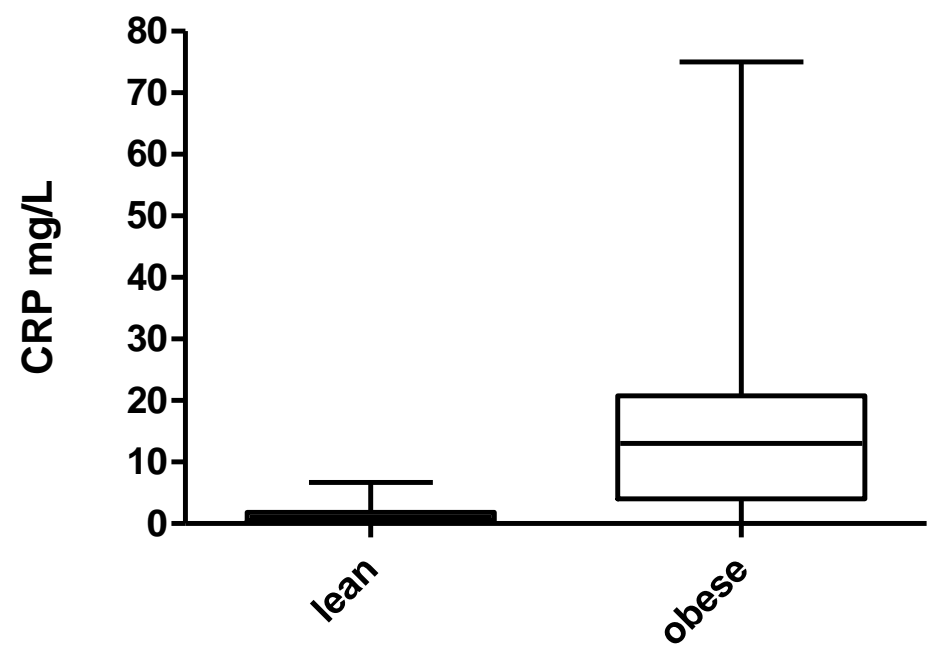


Click here to download Supplementary Files: Biomaterials Manuscript Informed trial 27062016 remarks.docx 
point-by-point response
Click here to download Supplementary Files: Reply to reviewers inFORMED.docx

point-by-point response
Click here to download Supplementary Files: Reply to reviewers inFORMED.docx

$x$

